\title{
Identification of an open crack in a beam with variable profile by two resonant frequencies
}

\author{
Lourdes Rubio*, José Fernández-Sáez ${ }^{\dagger}$ and Antonino Morassi ${ }^{\ddagger}$
}

June 28, 2016

\footnotetext{
${ }^{*}$ Corresponding author. Department of Mechanical Engineering, University Carlos III of Madrid, Avda. de la Universidad 30, 28911 Leganés, Madrid, Spain. Tel.: +34 91 6249403; fax: +3491 6249465. E-mail: Irubio@ing.uc3m.es

${ }^{\dagger}$ Department of Continuum Mechanics and Structural Analysis, University Carlos III of Madrid, Avda. de la Universidad 30, 28911 Leganés, Madrid, Spain. E-mail: ppfer@ing.uc3m.es

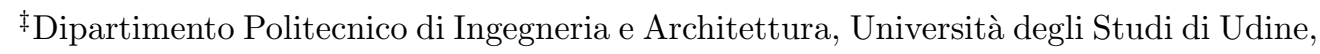
via Cotonificio 114, 33100 Udine, Italy. E-mail: antonino.morassi@uniud.it
} 


\begin{abstract}
We consider the identification of a single open crack in a simplysupported beam having non-uniform smooth profile and undergoing infinitesimal in-plane flexural vibration. The profile is assumed to be symmetric with respect to the mid-point of the beam axis. The crack is modelled by inserting a rotational linearly elastic spring at the damaged cross section. We establish sufficient conditions for the unique identification of the crack by a suitable pair of natural frequency data, and we present a constructive algorithm for determining the damage parameters. The result is proved under a technical a-priori assumption on the zeros of a suitable function determined in terms of the eigenfunctions of the problem. Extensions to beams under different sets of end conditions are also discussed. Theoretical results are confirmed by an extensive numerical investigation, both on simulated and experimental data.
\end{abstract}

Keywords: Damage identification, cracks, resonant frequencies, non-uniform beams, inverse problems

\title{
1 Introduction
}

In this paper we continue a line of research on dynamic methods for damage detection in structures initiated in Rubio et al. (2015) and aimed at the determination of a single open crack in one-dimensional beam elements with variable profile by minimal frequency data. 
A crucial point in damage identification is the modelling of the damage. Among various models that have been proposed in the literature to describe open cracks in beams, the localized flexibility model of cracks is the most common. In the case of beams under in-plane flexural vibration, the problem that we shall consider in this paper, an open crack is modelled by inserting a massless rotational elastic spring at the damaged cross-section, see, for example, Wendtland (1972), Freund and Herrmann (1976), Chondros and Dimarogonas (1980), Gudmundson (1983). The accuracy of the localized flexibility model has been evaluated in several vibration tests carried out on steel beams either with single or multiple cracks. The results confirm that modelling errors on low natural frequencies are comparable to those of the classical Euler-Bernoulli model for a beam without defects, see, for instance, Araujo Gomes and Montalvao Silva (1990), Caddemi and Morassi (2013). The localized flexibility model also provides an appreciable advantage in formulating the inverse problem. In fact, in the simplest case of a single crack, the unknown parameters correspond to the position $s$ of the elastic hinge and to the stiffness $K$ of the rotational spring (which may be related to the severity of the damage). This makes it reasonable to investigate to what extent the information on the crack-induced changes in a pair of natural frequencies can be useful for the identification of the damage.

A common approach to this issue consists in reformulating the inverse problem as an optimization problem. The damage parameters are estimated so that the first few natural frequencies (and, in some cases, also a set of eigenfunction amplitude values) closely match with the measured ones, see, among other contributions, the research developed by Shen and Taylor 
(1991), Davini et al. (1993), Vestroni and Capecchi (1996, 2000), Teughels et al. (2002), Sinha et al. (2002), Rubio (2009). The above identification methods allow to dealing with a large class of problems, such as, for example, crack detection in elastic frames (Morassi and Rovere (1997) and Greco and Pau (2012)), but they suffer the lack of general results. Basic questions such as how many data are necessary to ensure the uniqueness of the solution are rarely discussed in the literature and are still open.

Some general results on the identification of a single open crack in a bending vibrating beam are available when the beam is uniform and the crack is small. One of the first rigorous contributions to this inverse problem is due to Narkis (1994), who proved that a single small crack in a pinned-pinned uniform beam can be uniquely localized (up to a symmetric position) by the first two natural frequencies. Later on, working on the same problem, Morassi (2001) found closed-form expressions for $s$ and $K$ in terms of the frequency data, and extended Narkis's result to other suitable pairs of natural frequencies. Few years later, Dilena and Morassi (2004) proved that an appropriate use of natural frequencies and antiresonant frequencies can avoid the non-uniqueness of the damage location problem, which occurs, for example, in symmetrical beams when natural frequency data only are used. Key features of the method developed by Morassi and co-workers are, on the one side, the explicit expression of the eigenfrequency change induced by a small crack written in terms of known quantities of the undamaged configuration and, on the other side, the simple form taken by this expression for uniform beams under special set of boundary conditions. The methods by Narkis and Morassi are based on a perturbation analysis of the eigenvalue problem in a 
neighborhood of the undamaged beam, and are hardly applicable in the case of beams with variable profile.

A limited number of studies focussed on crack identification in nonuniform beams by frequency data. Among these, Nandwana and Maiti (1997) considered the identification of an open and no necessarily small crack in a piecewise-uniform cantilever. The procedure by Nandwana and Maiti has been subsequently extended by Chaudhari and Maiti (2000) to cantilever beams formed by two segments, one uniform in depth, the other segment with linearly varying depth, see also Chinchalkar (2001). The identification of multiple open cracks in a piecewise-constant beam under general boundary conditions was considered by Attar (2012) by using the exact expression of the characteristic equation of the damaged beam.

When the beam has generic variable profile the characteristic equation cannot be written in closed form and, at the best of our knowledge, no general results are available on the crack detection problem, even in the case of small damage. This open problem has been the motivation of our research and the present paper is a contribution to this issue.

In order to illustrate the main findings of our work, we refer, for the sake of clarity of presentation, to a simply-supported beam with variable profile. The profile is assumed to be smooth and symmetric with respect to the midpoint of the beam axis. Our analysis is based on three main steps. First, we show that the eigenvalue problem for the cracked beam can be transformed in an equivalent eigenvalue problem for a simply-supported beam carrying a point mass $m=\frac{1}{K}$ at the cracked cross-section $s$, with suitable bending stiffness and linear mass density coefficients (see Proposition 2.2 for a precise 
statement). Therefore, the crack detection problem is transformed into the equivalent problem of determining the location $s$ and magnitude $m$ of the point mass from a pair of natural frequencies. In the second step, we study the so-called $\lambda-m$ and $\lambda-s$ curves, that is the functions $\lambda_{n}=\lambda_{n}(s, \cdot)$ and $\lambda_{n}=\lambda_{n}(\cdot, m)$, for fixed $s$ and fixed $m$, respectively, where $\lambda_{n}$ is the $n$th eigenvalue, $n=1,2$ (see Proposition 4.2 and Theorem 4.5). The analysis is based on the explicit determination of the eigenvalue derivatives with respect to the parameters $s$ and $m$ (see Proposition 4.1) and on specific properties of the eigenpairs of the cracked beam. Under a technical a-priori assumption on the zeros of a suitable function determined in terms of the eigenfunctions of the problem, the above properties are used in the third and last step to define a constructive algorithm for solving the inverse problem (see Section 5). More precisely, it is shown that Narkis's result can be extended to nonuniform pinned-pinned beams, e.g., the crack can be uniquely determined, up to a symmetric position, from the knowledge of the first two natural frequencies of the beam.

For the sake of completeness, we comment the significant differences we have found in the present analysis and in the research recently developed by us in (Rubio et al., 2015) on the analogous inverse problem of detecting a single open crack in a longitudinally vibrating rod with variable profile from two natural frequencies. In the present case, two kind of additional difficulties arise. A first hindrance is connected with the study of qualitative properties of the eigenfunctions of the cracked beam, such as, the number of zeros and interlacing properties between the zeros of eigenfunctions and their derivatives. This study was carried out in Rubio et al. (2015) by 
extending classical Sturm-Liouville techniques for the undamaged rod to a rod with a crack. Sturm-Liouville methods are not easily extendable to fourth order operators, such as the Euler-Bernoulli operator governing the bending vibration of a cracked beam, and, therefore, we were forced to follow a different approach, mainly based on the study of the oscillatory character of the statical Green's function of the cracked beam. A second obstruction is connected with the study of the qualitative behavior of the $\lambda$-s curves and, particularly, with the determination of their stationarity points. It can be shown that the argument used by Rubio et al. (2015) does not apply to the fourth-order case. The technique we have adopted here is different and it is essentially based on a deformation argument which allowed us to reduce the analysis to the study of the zeros of a suitable function defined on the undamaged configuration (see the proof of Theorem 4.5). It is precisely at this point that, in order to apply the deformation argument, we have introduced the Vanishing Condition (20), that is an a-priori assumption on the zeros of a suitable function determined in terms of the eigenfunctions of the cracked beam. It can be shown that the hypothesis (20) is actually a property of the problem in the case of small damage (see Section 8).

The identification method has been tested on an extensive series of numerical simulations on beams having different profile and with various positions and severities of the crack. A selected set of numerical numerical results, both on simulated and experimental data, is presented and commented in Section 7. Numerical simulations performed even for severe levels of damage have not disproved the validity of our a-priori assumption, whose general validity, however, remains an open question. Finally, extension of the above 
results to beams under different set of end conditions is presented in Section 6.

\section{Formulation of the inverse problem and main result}

Let us consider a straight thin simply supported beam with variable profile under bending vibration. We assume that the beam has a single crack at the cross-section of abscissa $z_{d}$, with $0<z_{d}<L$, where $L$ is the length of the beam. The crack is assumed to remain open during the vibration and it is modelled as a massless rotational linearly elastic spring with stiffness $\widehat{K}$, see, for example, Freund and Herrmann (1976) for a justification of this localized flexibility model of crack based on Fracture Mechanics arguments, and to Caddemi and Morassi (2013) for an alternative derivation. The value of $\widehat{K}$ depends on the geometry of the cracked cross-section and on the material properties of the beam.

The free undamped bending vibrations of the beam with radian frequency $\omega$ and spatial amplitude $u=u(x)\left(x=\frac{z}{L}, z \in[0, L]\right)$ are governed by the following eigenvalue problem (written in dimensionless form)

$$
\left\{\begin{array}{l}
\left(a u^{\prime \prime}\right)^{\prime \prime}-\lambda \rho u=0, \quad \text { in }(0, s) \cup(s, 1), \\
u(0)=u^{\prime \prime}(0)=0, \\
{[[u(s)]]=\left[\left[\left(a u^{\prime \prime}\right)(s)\right]\right]=\left[\left[\left(a u^{\prime \prime}\right)^{\prime}(s)\right]\right]=0,} \\
K\left[\left[u^{\prime}(s)\right]\right]=a(s) u^{\prime \prime}(s), \\
u(1)=u^{\prime \prime}(1)=0,
\end{array}\right.
$$


where $s=\frac{z_{d}}{L}, s \in(0,1), K=\frac{\widehat{K} L}{E I_{0}}, K \in(0, \infty), \lambda=\frac{L^{4} \omega^{2}}{E I_{0}}$ and $I_{0}=C L^{4}$, with $C>0$ a suitable absolute constant. We use the notation $(\cdot)^{\prime}=\frac{d(\cdot)}{d x}$, $[[u(s)]]=$ $\left(\lim _{x \rightarrow s^{+}} u(x)-\lim _{x \rightarrow s^{-}} u(x)\right)$ to indicate $x$-differentiation and the jump of the function $u=u(x)$ at $x=s$, respectively. Here, $E$ is the (constant) Young's modulus of the material, $E>0 ; \rho=\rho(x)$ is the linear mass density; $a=a(x)$ is the (dimensionless) second moment of area about the axis through the centroid of the cross-section, at right angles to the plane of vibration (the neutral axis). We shall assume throughout that both $a(x)$ and $\rho(x)$ are strictly positive in $[0,1]$, i.e., there exist constants $a_{0}, \rho_{0}$ such that

$$
a(x) \geq a_{0}>0, \quad \rho(x) \geq \rho_{0}>0, \quad \text { in }[0,1]
$$

and twice continuously differentiable in $[0,1]$, i.e.,

$$
a \in C^{2}([0,1]), \quad \rho \in C^{2}([0,1]) .
$$

Under our assumptions, each eigenfunction $u$ is a continuous function in $[0,1]$ and belongs to $C^{4}((0, s) \cup(s, 1))$.

The main properties of the eigenpairs of (1)-(5) are stated in the following proposition.

Proposition 2.1. Under the above assumptions:

1. there exists a numerable sequence of real positive eigenvalues $\left\{\lambda_{n}\right\}_{n=1}^{\infty}$ of (1)-(5), with $\lim _{n \rightarrow \infty} \lambda_{n}=\infty$.

2. The eigenvalues $\left\{\lambda_{n}\right\}_{n=1}^{\infty}$ of (1)-(5) are all simple, e.g.,

$$
0<\lambda_{1}<\lambda_{2}<\ldots<\lambda_{n}<\ldots
$$


and to each eigenvalue $\lambda_{n}$ we can associate a unique eigenfunction, e.g., if $u_{n}$ and $\widetilde{u}_{n}$ are eigenfunctions associated to the same eigenvalue $\lambda_{n}$, then $u_{n}(x)=C \widetilde{u}_{n}(x)$ in $[0,1]$, where $C$ is a no-vanishing constant.

3. The set of eigenfunctions $\left\{u_{n}(x)\right\}_{n=1}^{\infty}$ forms an orthonormal basis of the space of continuous functions on $[0,1]$, vanishing at $x=0$ and $x=1$, with respect to the usual scalar product $<f, g\rangle=\int_{0}^{1} \rho f g$.

4. The $n$th eigenfunction $u_{n}(x)$ has exactly $(n-1)$ simple zeros in $(0,1)$, $n \geq 1$.

Properties 1. and 3. follow from general results for self-adjoint compact operators in Hilbert spaces. The arguments shown, for example, in Brezis (1986) can be adapted to obtain a proof of 1 . and 3.. Properties 2. and 4. (which do not hold, in general, for the Euler-Bernoulli operator) follows, for example, from the oscillatory character of the statical Green's function associated to (1)-(5), see the Appendix (subsection 10.1) for details.

We shall consider hereinafter symmetric beams in (1)-(5), that is beams for which the moment of inertia and the linear mass density are even functions with respect to the mid-point of the beam axis, e.g.,

$$
a(x)=a(1-x), \quad \rho(x)=\rho(1-x), \quad x \in[0,1] .
$$

In order to study the inverse problem of detecting a single crack from a pair of natural frequencies of (1)-(5), we found convenient reformulate (1)(5) as an equivalent eigenvalue problem. The equivalence is stated in the next proposition.

Let $u$ be a non-trivial solution of (1)-(5) associated to an eigenvalue $\lambda$.

\section{Proposition 2.2.}


i) Let $(\lambda, u)$ be an eigenpair of (1)-(5). Then $\lambda$ is an eigenvalue of the problem

$$
\left\{\begin{array}{l}
\left(b v^{\prime \prime}\right)^{\prime \prime}-\lambda r v=0, \quad \text { in }(0, s) \cup(s, 1), \\
v(0)=v^{\prime \prime}(0)=0, \\
{[[v(s)]]=\left[\left[v^{\prime}(s)\right]\right]=\left[\left[\left(b v^{\prime \prime}\right)(s)\right]\right]=0} \\
{\left[\left[\left(b v^{\prime \prime}\right)^{\prime}(s)\right]\right]=\lambda m v(s)} \\
v(1)=v^{\prime \prime}(1)=0
\end{array}\right.
$$

where

$v=-a u^{\prime \prime} \quad$ in $(0, s) \cup(s, 1), \quad b=\rho^{-1}$ and $r=a^{-1}$ in $\in[0,1], \quad m=K^{-1}$.

ii) Conversely, let $(\lambda, v)$ be an eigenpair of (10)-(14). Then $\lambda$ is an eigenvalue of the problem (1)-(5) with

$$
u=-b v^{\prime \prime} \quad \text { in }(0, s) \cup(s, 1), \quad a=r^{-1} \text { and } \rho=b^{-1} \text { in }[0,1], \quad K=m^{-1} \text {. }
$$

Proof. Let us consider the statement i), the proof of ii) being similar. Using (15) in (1), dividing by $\rho$ and differentiating twice, we obtain (10). The end conditions $v(0)=v(1)=0$ and the jump conditions $[[v(s)]]=\left[\left[v^{\prime}(s)\right]\right]=0$ follow directly by the corresponding end and jump conditions of (1)-(5). The end conditions $v^{\prime \prime}(0)=v^{\prime \prime}(1)=0$ and the jump condition $\left[\left[\left(b v^{\prime \prime}\right)(s)\right]\right]=0$ can be deduced by using the differential equation (1) to determine the limit values of $v^{\prime \prime}$ at $x=0^{+}, x=1^{-}, x=s^{ \pm}$. Finally, inserting $v=-a u^{\prime \prime}$ in (1), dividing by $\rho$, differentiating and using (4), one can deduce the jump condition (13). 
Remark 2.3. Problem (10)-(14) describes the free bending vibration of a beam simply supported at the ends, with bending stiffness $b$ and linear mass density $r$, carrying a point mass $m$ at $x=s$. Note that, by properties (6)-(7) of the coefficients $a$ and $\rho$, we have

$$
b(x) \geq b_{0}>0, \quad r(x) \geq r_{0}>0, \quad b \in C^{2}([0,1]), \quad r \in C^{2}([0,1]),
$$

for some constants $b_{0}, r_{0}$. Moreover, after reduction to the equivalent problem by Proposition 2.2, the functions $b=b(x)$ and $r=r(x)$ still remain even functions with respect to the mid-point of the beam axis, e.g.,

$$
b(x)=b(1-x), \quad r(x)=r(1-x), \quad x \in[0,1] .
$$

Remark 2.4. Basing on the equivalence between the eigenvalue problems (1)-(5) and (10)-(14) stated in Proposition 2.2, in the following we shall be mainly concerned with the formulation in terms of the vibration of the beam with the point mass. The functional space suitable for (10)-(14) is made by functions more regular than those occurring in (1)-(5), e.g., the jump of the first derivative $v^{\prime}$ at $x=s$ is not allowed, whereas $u^{\prime}$ may be discontinuous at the crack location. This additional regularity simplifies the study of the dependence of an eigenvalue on the damage parameters $s$ and $K$ (or $m$ ), see Section 4.

Let $\left(\lambda_{n}, v_{n}(x ; s, m)\right)$ be the $n$th eigenpair of (10)-(14). For every $m$, $m \geq 0$, let us introduce the function

$$
f_{m}^{(n)}:[0,1] \rightarrow \mathbb{R}, \quad f_{m}^{(n)}(s)=\left.\left(v_{n}(x ; s, m) \frac{d v_{n}(x ; s, m)}{d x}\right)\right|_{x=s}, \quad n=1,2 .
$$

In the sequel we shall consider cracked beams such that, for $n=1,2$, and for 
every $m>0$, the function

$f_{m}^{(n)}=f_{m}^{(n)}(s)$ has a finite number of zeros in $[0,1]$, all of which are simple.

We call (20) the Vanishing Condition for $f_{m}^{(n)}$.

Remark 2.5. It is possible to show that the Vanishing Condition (20) is in fact a property satisfied by the eigensolutions of the problem (10)-(14), provided that the mass intensity $m$ is small enough, i.e., in the case of small crack. We refer to Section 8 for a proof.

We are now in position to state our main result.

Theorem 2.6. Under the above assumptions, the measurement of the first two natural frequencies of (10)-(14) allows for the unique determination of the intensity $m$ and the location $s$ of the point mass, up to the symmetric position $1-s$. The identification procedure is constructive.

Basing on the equivalence stated in Proposition 2.2, a direct consequence of the above theorem is the following result.

Corollary 2.7. Under the assumptions of Theorem 2.6, the measurement of the first two natural frequencies of (1)-(5) allows for the unique determination of the severity of the damage $K$ and the location $s$ of the crack, up to the symmetric position $1-s$. The identification procedure is constructive. 


\section{Some properties of the equivalent eigen- value problem}

The next proposition states some properties of the zeros of the first and second eigenfunction of (10)-(14) which will be useful in our analysis. For reader's convenience, details of a proof are collected in the Appendix (subsection 10.2).

Proposition 3.1. Let $v_{n}(x)$ be the $n$th eigenfunction of (10)-(14). Under the above assumptions:

1. $v_{1}(x)$ has no zeros in $(0,1)$ and $v_{1}^{\prime}(x)$ has exactly one simple zero in $(0,1)$.

2. $v_{2}(x)$ has exactly one simple zero in $(0,1)$, say at $\xi_{1}$, and $v_{2}^{\prime}(x)$ has exactly two simple zeros in $(0,1)$, say at $\eta_{1}, \eta_{2}$. Moreover, $\eta_{1}<\xi_{1}<\eta_{2}$.

Weak and variational formulation of the eigenvalue problem (10)-(14) will be used throughout the paper. Let us denote by $\mathcal{H}$ the functional space of admissible deformations of the beam:

$$
\mathcal{H}=\left\{f \mid f \in H^{2}(0,1), f(0)=f(1)=0\right\},
$$

where $H^{2}(0,1)$ is the Hilbert space of Lebesgue measurable functions $f$ : $(0,1) \rightarrow \mathbb{R}$ such that $f$, and its first and second weak derivatives are square summable in $(0,1)$, e.g., $\int_{0}^{1}\left(f^{2}+\left(f^{\prime}\right)^{2}+\left(f^{\prime \prime}\right)^{2}\right)<+\infty$.

The weak formulation of (10)-(14) consists in finding $v \in \mathcal{H} \backslash\{0\}$ such that

$$
\int_{0}^{1} b v^{\prime \prime} \varphi^{\prime \prime}=\lambda\left(m v(s) \varphi(s)+\int_{0}^{1} r v \varphi\right), \quad \text { for every } \varphi \in \mathcal{H}
$$


The Rayleigh's Quotient associated to (22) is

$$
R[\cdot]: \mathcal{H} \backslash\{0\} \rightarrow \mathbb{R}, \quad R[\varphi]=\frac{\int_{0}^{1} b\left(\varphi^{\prime \prime}\right)^{2}}{m \varphi^{2}(s)+\int_{0}^{1} r \varphi^{2}} .
$$

The eigenvalues can be determined by solving the chain of minimum problems

$$
R\left[v_{n}\right]=\min _{\varphi \in V_{n} \backslash\{0\}} R[\varphi]=\lambda_{n}
$$

where

$$
V_{n}=\left\{\varphi \in \mathcal{H} \text { s.t. } m v_{i}(s) \varphi(s)+\int_{0}^{1} r v_{i} \varphi=0, i=1, \ldots, n-1\right\} .
$$

An equivalent formulation follows from the Maximum-Minimum Principle for the eigenvalues of (10)-(14), namely

$$
\lambda_{n}=\max _{l_{i} \in \mathcal{H}^{\prime}, i=1, \ldots, n-1}\left\{\min _{\varphi \in \mathcal{H} \backslash\{0\}, l_{i}(\varphi)=0, i=1, \ldots, n-1} R[\varphi]\right\},
$$

where $\mathcal{H}^{\prime}$ is the dual space of $\mathcal{H}$, that is the space of all the linear and continuous real-valued functionals $l_{i}$ on $\mathcal{H}$. We refer to Weinberger (1965) for a complete account of the above formulations.

In the next sections we shall often compare the eigenpairs of the problem (10)-(14) for finite, no-vanishing $m$, and $s \in(0,1)$, to those obtained by taking either $m=0$ or $\{s=0, s=1\}$ in (10)-(14). We shall denote by $\left(\lambda_{n}^{U}, v_{n}^{U}\right)$ the $n$th eigenpair of the unperturbed problem

$$
\left\{\begin{array}{l}
\left(b v^{U^{\prime \prime}}\right)^{\prime \prime}-\lambda^{U} r v^{U}=0, \quad x \in(0,1), \\
v^{U}(0)=v^{U^{\prime \prime}}(0)=0 \\
v^{U}(1)=v^{U^{\prime \prime}}(1)=0
\end{array}\right.
$$

By analogy, the eigenpairs $\left\{\left(\lambda_{n}, v_{n}\right)\right\}_{n=1}^{\infty}$ of the problem (10)-(14) will also be called perturbed eigenpairs. There exists a numerable sequence of real, 
simple, strictly positive eigenvalues of (27)-(29), say $0<\lambda_{1}^{U}<\lambda_{2}^{U}<\ldots$, with $\lim _{n \rightarrow \infty} \lambda_{n}^{U}=+\infty$, and Proposition 3.1 is well-known when $m=0$ (see, for example, Gladwell (2004)). Weak, variational and Maximum-Minimum formulation of (27)-(29) can be deduced by the corresponding formulations (22), (24)-(25) and (26), respectively, taking formally $m=0$.

Variational and Maximum-Minimum formulations are useful to derive the following bounds, see, for example, Courant and Hilbert (1966; Vol. 1, Chapter VI, Paragraph 2).

Proposition 3.2. Under the above assumptions and notation, we have

$$
\lambda_{n-1}^{U} \leq \lambda_{n} \leq \lambda_{n}^{U}, \quad \text { for every } n \geq 1,
$$

where we have defined $\lambda_{0}^{U}=0$.

The right inequality in (30) (upper bound of $\lambda_{n}$ ) shows that the addition of a mass $m$ decreases the natural frequencies of the unperturbed beam, or, in other words, that a crack decreases natural frequencies. The left inequality in (30) (lower bound of $\lambda_{n}$ ) follows as in any constrained system by adding the linear constraint $v(s)=0$ to the perturbed system having a point mass at $s$ or, equivalently, by assuming that the bending moment vanishes at the crack location in equations (1)-(5).

\section{$4 \lambda-\mathrm{m}$ and $\lambda$-s curves}

In order to extract quantitative information on the identification parameters $m$ and $s$ from the eigenvalues, in the following proposition we shall introduce the first-order derivatives of an eigenvalue of (10)-(14) with respect to $m$ and 
$s$. For given coefficients $b$ and $r$, an eigenpair $(\lambda, v)$ of (10)-(14) depends on the perturbation parameters $s$ and $m$. When necessary, we shall explicitly show this dependence by writing $\lambda=\lambda(s, m)$ and $v=v(x ; s, m)$, where $x \in[0,1]$ is the current spatial variable.

Proposition 4.1. Let $(\lambda, v)$ be an eigenpair of (10)-(14). We have

$$
\begin{gathered}
\frac{\partial \lambda}{\partial s}=-2 \lambda \frac{m v(s) v^{\prime}(s)}{m v^{2}(s)+\int_{0}^{1} r v^{2}}, \\
\frac{\partial \lambda}{\partial m}=-\lambda \frac{v^{2}(s)}{m v^{2}(s)+\int_{0}^{1} r v^{2}},
\end{gathered}
$$

where $v(s)=\left.v(x ; s, m)\right|_{x=s}, v^{\prime}(s)=\left.\left(\frac{d v(x ; s, m)}{d x}\right)\right|_{x=s}$.

The proof of the proposition can be obtained by adapting the ideas of the proof of the analogous property for a cracked beam under axial vibration, see Proposition 4.1 in Rubio et al. (2015).

We shall now study the so-called $\lambda$-m and $\lambda$-s curves, that is the functions $\lambda_{n}=\lambda_{n}(s, \cdot), \lambda_{n}=\lambda_{n}(\cdot, m), n=1,2$. This analysis will be useful in implementing the identification algorithm of Section 5 .

We begin with the study of the dependence of $\lambda_{n}$ on the parameter $m$, for a given position $s$ of the point mass.

Proposition 4.2. Let $\left(\lambda_{n}, v_{n}\right),\left(\lambda_{n}^{U}, v_{n}^{U}\right)$ be the $n$th eigenpair of the problem (10)-(14), (27)-(29), respectively, $n=1,2$.

i) If $v_{n}^{U}\left(s_{0}\right)=0$ for some $s_{0} \in[0,1]$, then $\lambda_{n}\left(s_{0}, m\right)=\lambda_{n}^{U}$ for every finite positive $m$.

ii) If $v_{n}^{U}\left(s_{0}\right) \neq 0$ for some $s_{0} \in(0,1)$, then $\lambda_{n}=\lambda_{n}\left(s_{0}, m\right)$ is a monotonically decreasing function of $m$ in $[0, \infty)$. 
iii) If $\lambda_{n}\left(s_{0}, m_{0}\right)=\lambda_{n}^{U}$ for some $s_{0} \in[0,1]$ and $m_{0} \in(0, \infty)$, then $v_{n}^{U}\left(s_{0}\right)=0$.

iv) If $v_{n}\left(s_{0} ; s_{0}, m_{0}\right)=0$ for some $s_{0} \in[0,1]$ and $m_{0} \in(0, \infty)$, then $v_{n}^{U}\left(s_{0}\right)=$ 0 .

A proof of the proposition can be obtained following the lines of the proof of the analogous property for a cracked beam under axial vibration, see Proposition 5.1 in Rubio et al. (2015).

A simple consequence of the symmetry (18) of the coefficients $b$ and $r$ on the eigenpairs of the problem (10)-(14) is the following property.

Proposition 4.3. Let $\left(\lambda_{n}, v_{n}\right)$ be the $n$th eigenpair of (10)-(14) for coefficients $b$ and $r$ satisfying (17) and (18), $n \geq 1$. Let $m$ be given, $0<m<\infty$. Then

$$
\lambda_{n}(s)=\lambda_{n}(1-s), \quad s \in[0,1] .
$$

If $s=\frac{1}{2}$, then

$$
\text { for } n \text { odd, we have } v_{n}(x)=v_{n}(1-x) \text {, }
$$

and

$$
\text { for } n \text { even, we have } v_{n}(x)=-v_{n}(1-x), \quad x \in[0,1] \text {. }
$$

The above statements can be easily verified by direct calculation. We notice the following simple result.

Corollary 4.4. Under the assumptions of Proposition 4.3, for every $n \geq 1$, we have

$$
\frac{\partial \lambda_{n}}{\partial s}(s)=0 \quad \text { for } s \in\left\{0,1, \frac{1}{2}\right\}
$$

The identification method described in Section 5 is based on the following key result, which is presented for the first two eigenvalues of (10)-(14) only, since solely them are used to formulate and solve our inverse problem. 
Theorem 4.5. Let $\left(\lambda_{n}, v_{n}\right), n=1,2$, be the $n$th eigenpair of (10)-(14) for coefficients $b$ and $r$ satisfying (17) and (18). Assume that the Vanishing Condition (20) is satisfied. Then, for a given $m, m>0$, we have:

i) $\lambda_{1}=\lambda_{1}(s)$ is a strictly decreasing function in $\left(0, \frac{1}{2}\right)$;

ii) there exists a unique $\widetilde{s} \in\left(0, \frac{1}{2}\right)$ such that $\frac{\partial \lambda_{2}}{\partial s}(\widetilde{s})=0$, that is $\lambda_{2}=\lambda_{2}(s)$ is a strictly decreasing function and a strictly increasing function in $(0, \widetilde{s})$ and in $\left(\widetilde{s}, \frac{1}{2}\right)$, respectively.

For a proof of Theorem 4.5 we shall make use of the following Deformation Lemma.

Lemma 4.6. Let $f_{t}=f_{t}(x)$ be at-family of real-valued functions of $x \in[0,1]$ which are continuous and jointly continuously differentiable in $x$ and in $t$, where the parameter $t$ belongs to the interval $\left[t_{1}, t_{2}\right],-\infty<t_{1}<t_{2}<+\infty$. Suppose that for every $t \in\left[t_{1}, t_{2}\right]$, the function $f_{t}$ has a finite number of zeros in $[0,1]$, all of which are simple, and has boundary values at $x=0$ and $x=1$ that are independent of $t$. Then, the number of zeros of $f_{t}$ is independent of $t$, for all $t$ satisfying $t_{1} \leq t \leq t_{2}$.

This lemma has been introduced in Pöschel and Trubowitz (1987, p. 41).

Proof of Theorem 4.5. Case i). By the expression (31) of the partial derivative of $\lambda_{1}$ with respect to the mass position $s$, the sign of $\frac{\partial \lambda_{1}}{\partial s}$ depends on the sign of the product $\left.\left(v_{1}(x ; s, m) \frac{d v_{1}(x ; s, m)}{d x}\right)\right|_{x=s}$. Let $M>0$ be a finite number. For every $m, m \in[0, M]$, let us introduce the function

$$
f_{m}^{(1)}:[0,1] \rightarrow \mathbb{R}, \quad f_{m}^{(1)}(s)=\left.\left(v_{1}(x ; s, m) \frac{d v_{1}(x ; s, m)}{d x}\right)\right|_{x=s} .
$$

By the regularity of the eigenfunction $v_{1}$ with respect to $s$ and $m$, the function $f_{m}^{(1)}$ is continuous and jointly differentiable in $s$ and $m$, for every $s \in[0,1]$ 
and every $m \in[0, M]$. The function $f_{m}^{(1)}$ vanishes identically at $s=0$ and $s=1$, for every $m \in[0, M]$. Therefore, under the Vanishing Condition (20), the function $f_{m}^{(1)}$ satisfies all the conditions required by Lemma 4.6 to the function $f_{t}$, where the variables $t$ and $x$ are replaced by $m$ and $s$, respectively. It follows that, for every $m \in[0, M]$, the number of zeros of $f_{m}^{(1)}$ is equal to the number of zeros of $f_{0}^{(1)}$. Noticing that for $m=0$ we have $v_{1}(s ; s, m=0)=v_{1}^{U}(s)$, namely the eigenfunction $v_{1}$ coincides with the unperturbed eigenfunction $v_{1}^{U}$, we have

$$
f_{0}^{(1)}(s)=v_{1}^{U}(s)\left(v_{1}^{U}(s)\right)^{\prime}, \quad s \in[0,1]
$$

By standard results (or, equivalently, by Proposition 3.1 with $m=0$ ), $v_{1}^{U}$ has one sign in $(0,1)$ and $\left(v_{1}^{U}\right)^{\prime}$ has a single simple zero in $(0,1)$, precisely at $s=\frac{1}{2}$ because of the symmetry of the beam. Therefore, $f_{m}^{(1)}$ has only one simple zero in $(0,1)$, exactly at $s=\frac{1}{2}$, for every $m \in[0, M]$. Finally, taking into account that $0<\lambda_{1}(s) \leq \lambda_{1}^{U}$ in $[0,1]$ (by Proposition 3.2) and $\lambda_{1}\left(\frac{1}{2}\right)<\lambda_{1}^{U}$ (since $v_{1}^{U}\left(\frac{1}{2}\right) \neq 0$, see Proposition 4.2), we can conclude that $s=\frac{1}{2}$ is the only zero of $\frac{\partial \lambda_{1}}{\partial s}$ in $(0,1)$. By the arbitrariness of $M$, the thesis follows.

Case ii). The proof for $n=2$ follows the same lines of the proof of case i). In brief, under our assumptions, the family of functions

$$
f_{m}^{(2)}:[0,1] \rightarrow \mathbb{R}, \quad f_{m}^{(2)}(s)=\left.\left(v_{2}(x ; s, m) \frac{d v_{2}(x ; s, m)}{d x}\right)\right|_{x=s},
$$

for $s \in[0,1]$ and $m \in[0, M]$, satisfies the assumptions required in Lemma 4.6. Moreover, for $m=0$, by Proposition 3.1 and Proposition 4.3, the function

$$
f_{0}^{(2)}(s)=v_{2}^{U}(s)\left(v_{2}^{U}(s)\right)^{\prime}
$$


has exactly three simple zeros in $(0,1)$, namely at $\frac{1}{2}, \eta_{1} \in\left(0, \frac{1}{2}\right)$ and $\eta_{2}=$ $1-\eta_{1} \in\left(\frac{1}{2}, 1\right)$. Then, by Lemma 4.6 , the function $f_{m}^{(2)}$ has exactly three simple zeros in $(0,1)$ for every $m \in[0, M]$. By the symmetry of the beam, $f_{m}^{(2)}$ vanishes at $\frac{1}{2}$, and the remaining two simple zeros are located at $\widetilde{s} \in\left(0, \frac{1}{2}\right)$ and at $(1-\widetilde{s}) \in\left(\frac{1}{2}, 1\right)$. Note that $\widetilde{s}$ generally depends on $m$. Finally, recalling that $\lambda_{2}(s) \leq \lambda_{2}^{U}$ in $[0,1]$ (by Proposition 3.2) and that $\lambda_{2}\left(\frac{1}{2}\right)=\lambda_{2}^{U}$ for every $m$ (since $v_{2}^{U}\left(\frac{1}{2}\right)=0$, see Proposition 4.2), the thesis follows.

\section{A constructive identification algorithm}

In this section we prove Theorem 2.6 by presenting a constructive algorithm for the determination of the parameters $\{s, m\}$ in the problem (10)-(14) (or, equivalently, the position of the crack $s$ and its severity $K$ in (1)-(5)) from the knowledge of the first two eigenvalues, say $\bar{\lambda}_{1}, \bar{\lambda}_{2}$.

We recall that the beam coefficients $b$ and $r$ satisfy (17) and (18). Let $s \in(0,1)$ and $m \in(0, \infty)$.

By Proposition 3.2, input data $\left\{\bar{\lambda}_{1}, \bar{\lambda}_{2}\right\}$ are chosen such that

$$
0<\bar{\lambda}_{1}<\lambda_{1}^{U}, \quad \lambda_{1}^{U} \leq \bar{\lambda}_{2} \leq \lambda_{2}^{U}
$$

Note that, by Proposition 4.2 and Proposition 3.1, the upper bound for $\bar{\lambda}_{1}$ is strict, namely the first eigenvalue is always 'sensitive' to the point mass $m$.

If $\bar{\lambda}_{2}=\lambda_{2}^{U}$, then, by Proposition 4.2 and Proposition 4.3, the point mass is located at $s=\frac{1}{2}$. By Proposition $4.2, \lambda_{1}=\lambda_{1}\left(\frac{1}{2}, m\right)$ is a monotonically decreasing function of $m$ and, in addition, one can prove that

$$
\lim _{m \rightarrow \infty} \lambda_{1}\left(\frac{1}{2}, m\right)=0^{+}
$$


Then, by (42) and by the monotonicity of the function $\lambda_{1}\left(\frac{1}{2}, m\right)$ for $m \in$ $(0, \infty)$, we can uniquely determine $m$ by solving the equation $\bar{\lambda}_{1}=\lambda_{1}\left(\frac{1}{2}, m\right)$.

In the remaining of the section we shall consider the non-trivial condition $\bar{\lambda}_{2}<\lambda_{2}^{U}$ and, by symmetry hypothesis (see Proposition 4.3), we shall assume $s \in\left(0, \frac{1}{2}\right)$.

Basing on the properties of the $\lambda-\mathrm{m}$ and $\lambda$-s curves stated in Section 4, it can be shown that the algorithm developed in Rubio et al. (2015) for the identification of a point mass in a longitudinally vibrating rod can be adapted to the present case. Therefore, in the sequel we shall simply show the main steps of the identification procedure, referring to the above mentioned paper for details on the convergence of the method to the actual solution.

The flow-chart of the identification procedure is as follows.

1. Let $\bar{\lambda}_{1}, \bar{\lambda}_{2}$ be the measured values of the first and second eigenvalue, respectively, with $\bar{\lambda}_{1}<\lambda_{1}^{U}$ and $\lambda_{1}^{U} \leq \bar{\lambda}_{2}<\lambda_{2}^{U}$.

2. We determine the values $m_{1}^{-}, m_{2}^{-}, 0<m_{i}^{-}<\infty, i=1,2$, of the parameter $m$ such that

$$
\bar{\lambda}_{1}=\lambda_{1}\left(\frac{1}{2}, m_{1}^{-}\right), \quad \bar{\lambda}_{2}=\lambda_{2}\left(s_{2 m i n}, m_{2}^{-}\right),
$$

where $s_{2 \min } \in\left(0, \frac{1}{2}\right)$ is the unique point such that $\left.\frac{\partial \lambda_{2}\left(s, m_{2}^{-}\right)}{\partial s}\right|_{s=s_{2 \min }}=0$ (see Theorem 4.5 , point ii)). Note that $m_{1}^{-} \neq m_{2}^{-}$and

$$
\max \left\{m_{1}^{-}, m_{2}^{-}\right\}<m \text {. }
$$

3. We distinguish two main cases.

CASE 1. If

$$
\max \left\{m_{1}^{-}, m_{2}^{-}\right\}=m_{1}^{-},
$$


then we determine the curve $y=\lambda_{2}\left(s, m_{1}^{-}\right)$in $[0,1]$, see Figure 1 . Let us consider the curves $y=\lambda_{2}(s, \mathcal{M})$ for $\mathcal{M}>m_{1}^{-}, \mathcal{M}$ not too large. Let us denote by $P_{2 r}(\mathcal{M})$ the intersection point between $y=\lambda_{2}(s, \mathcal{M})$ and $y=\bar{\lambda}_{2}$, with the abscissa $s\left(P_{2 r}(\mathcal{M})\right)$ such that $s\left(P_{2 r}(\mathcal{M})\right)>s_{2 m i n}$. Moreover, let us denote by $P_{1}(\mathcal{M})$ the unique intersection point between $y=\lambda_{1}(s, \mathcal{M})$ and $y=\bar{\lambda}_{1}$, with $s\left(P_{1}(\mathcal{M})\right)<\frac{1}{2}$. Then, basing on Proposition 4.2 and Theorem 4.5, it can be proved that there exists a unique value of $\mathcal{M}$, say $\widetilde{\mathcal{M}}$, such that $s\left(P_{2 r}(\widetilde{\mathcal{M}})\right)=s\left(P_{1}(\widetilde{\mathcal{M}})\right)$. The value $\widetilde{\mathcal{M}}$ is the intensity of the mass $m$ and $s=s\left(P_{1}(\widetilde{\mathcal{M}})\right)$ is its position.

CASE 2. If

$$
\max \left\{m_{1}^{-}, m_{2}^{-}\right\}=m_{2}^{-},
$$

we determine the curve $y=\lambda_{1}\left(s, m_{2}^{-}\right)$, denoting by $P_{1}\left(m_{2}^{-}\right)$the unique intersection point between $y=\lambda_{1}\left(s, m_{2}^{-}\right)$and $y=\bar{\lambda}_{1}$, with abscissa $s_{1}=s\left(P_{1}\left(m_{2}^{-}\right)\right) \in\left(0, \frac{1}{2}\right)$, see Figure 2.

At this stage, we distinguish two additional subcases.

Case 2. - a): Assume that

$$
s_{2 \min } \leq s_{1}
$$

If $s_{2 \min }=s_{1}$, then the problem is solved. If $s_{2 m i n}<s_{1}$, we can repeat the procedure used in CASE 1, and the inverse problem has a unique solution, see Figure 2.

CASE 2. - b): If

$$
s_{2 \min }>s_{1}
$$


then there exists $m^{*}>m_{2}^{-}$such that the intersection point $P_{2 l}\left(m^{*}\right)$ between $y=\lambda_{2}\left(s, m^{*}\right)$ and $y=\bar{\lambda}_{2}$ satisfies $s\left(P_{2 l}\left(m^{*}\right)\right)<s\left(P_{1}\left(m_{2}^{-}\right)\right)$, where $P_{1}\left(m_{2}^{-}\right)$is the unique intersection point between $y=\lambda_{1}\left(s, m_{2}^{-}\right)$ and $y=\bar{\lambda}_{1}$, see Figure 3. By decreasing the mass value from $m^{*}$ to $m_{2}^{-}$, there exists a unique value, say $\widetilde{\mathcal{M}}$, such that $s\left(P_{2 l}(\widetilde{\mathcal{M}})\right)=s\left(P_{1}(\widetilde{\mathcal{M}})\right)$, and the identified parameters are $m=\widetilde{\mathcal{M}}$ and $s=s\left(P_{1}(\widetilde{\mathcal{M}})\right)$.

Applications and details on a selected set of numerical simulations are reported in Section 7.

\section{Extensions}

In this section we show how the method presented in previous sections can be extended to cover other sets of end conditions (Section 6.1), and how it can be improved to determine a unique solution of the inverse problem from two suitable frequency data (Section 6.2). Proof of these results are based on the behavior of the $\lambda$-m and $\lambda$-s curves for the corresponding eigenvalue problems. In turn, these properties follow from qualitative results of the eigensolutions analogous to those presented in previous sections. Since most of the steps in the preceding proofs can be duplicated, in the sequel we shall simply state the main results useful for our analysis, leaving the details aside.

\subsection{Crack identification in a free-free beam}

Let us consider the cracked beam introduced at the beginning of Section 2, with coefficients $a, \rho$ satisfying (6), (7), and with a single open crack of severity $K, K \in(0, \infty)$, at the position $s, s \in(0,1)$. Assume that both ends of 
the beam are free. It should be noted that this set of end conditions is rather common in experimental investigations, since it avoids the introduction of uncertainty connected with boundary condition modelling, see, for example, Araujo Gomes and Montalvao Silva (1990) and Caddemi and Morassi (2013). The free undamped bending vibrations of the cracked beam are governed by the following eigenvalue problem (in dimensionless form)

$$
\left\{\begin{array}{l}
\left(a u^{\prime \prime}\right)^{\prime \prime}-\lambda \rho u=0, \quad \text { in }(0, s) \cup(s, 1), \\
\left(a u^{\prime \prime}\right)(0)=\left(a u^{\prime \prime}\right)^{\prime}(0)=0 \\
{[[u(s)]]=\left[\left[\left(a u^{\prime \prime}\right)(s)\right]\right]=\left[\left[\left(a u^{\prime \prime}\right)^{\prime}(s)\right]\right]=0} \\
K\left[\left[u^{\prime}(s)\right]\right]=a(s) u^{\prime \prime}(s) \\
\left(a u^{\prime \prime}\right)(1)=\left(a u^{\prime \prime}\right)^{\prime}(1)=0
\end{array}\right.
$$

The eigenpairs of (49)-(53) are still denoted by $\left\{\left(\lambda_{n}, u_{n}\right)\right\}_{n=0}^{\infty}$, with $0=\lambda_{0}<$ $\lambda_{1}<\lambda_{2}<\ldots, \lim _{n \rightarrow \infty} \lambda_{n}=\infty$. The first eigenvalue has double multiplicity and the corresponding eigenspace is spanned by the two eigenfunctions $\{1, x\}$. By adapting the proof of Proposition 2.2, it can be seen that the positive eigenvalues of (49)-(53) coincide with the eigenvalues of a clamped-clamped beam carrying a point mass $m=K^{-1}$ at $x=s$ :

$$
\left\{\begin{array}{l}
\left(b v^{\prime \prime}\right)^{\prime \prime}-\lambda r v=0, \quad \text { in }(0, s) \cup(s, 1), \\
v(0)=v^{\prime}(0)=0, \\
{[[v(s)]]=\left[\left[v^{\prime}(s)\right]\right]=\left[\left[\left(b v^{\prime \prime}\right)(s)\right]\right]=0} \\
{\left[\left[\left(b v^{\prime \prime}\right)^{\prime}(s)\right]\right]=\lambda m v(s)} \\
v(1)=v^{\prime}(1)=0
\end{array}\right.
$$

where, analogously to $(15), v=-a u^{\prime \prime}$ in $(0, s) \cup(s, 1)$, and the coefficients $b=\rho^{-1}, r=a^{-1}$ satisfy (17). 
A direct inspection of the proofs of previous results shows that Proposition 3.1 (qualitative properties of the zeros of the eigenfunctions), Proposition 4.1 (eigenvalue derivatives) and Proposition 4.2 (behavior of the $\lambda-\mathrm{m}$ curves) can be suitably extended also to cover the set of free-free end conditions.

Hereinafter, we shall assume that the coefficients $b$ and $r$ satisfy the symmetry conditions (18). Therefore, the statements of Proposition 4.3 and Corollary 4.4 hold for the eigenpairs of the free-free beam.

For every $m, m \geq 0$, we assume that the Vanishing Condition (20) is satisfied by the eigenfunctions $v_{n}=v_{n}(x ; s, m), n=1,2$, of the problem (54)-(58). As for the pinned-pinned end conditions, it is possible to show that this Vanishing Condition actually is a property of the eigensolutions of the problem (54)-(58) for small mass intensity $m$, i.e., in the case of small crack.

The analogue of Theorem 4.5 is the following result.

Theorem 6.1. Let $\left(\lambda_{n}, v_{n}\right), n=1,2$, be the $n$th eigenpair of (54)-(58) for coefficients b, $r$ satisfying (17) and (18). Under the above assumptions, for every $m, m \in(0, \infty)$, we have:

i) $\lambda_{1}=\lambda_{1}(s)$ is a strictly decreasing function of $s$ in $\left(0, \frac{1}{2}\right)$, with $\lambda_{1}(0)=$ $\lambda_{1}(1)=\lambda_{1}^{U}, \lambda_{1}\left(\frac{1}{2}\right)<\lambda_{1}^{U}, \frac{\partial \lambda_{1}}{\partial s}(0)=\frac{\partial \lambda_{1}}{\partial s}\left(\frac{1}{2}\right)=\frac{\partial \lambda_{1}}{\partial s}(1)=0 ;$

ii) there exists a unique $\widetilde{s} \in\left(0, \frac{1}{2}\right)$ such that $\frac{\partial \lambda_{2}}{\partial s}(\widetilde{s})=0$, with $\lambda_{2}(\widetilde{s})<\lambda_{2}^{U}$; moreover, $\lambda_{2}(0)=\lambda_{2}\left(\frac{1}{2}\right)=\lambda_{2}(1)=\lambda_{2}^{U}, \frac{\partial \lambda_{2}}{\partial s}(0)=\frac{\partial \lambda_{2}}{\partial s}\left(\frac{1}{2}\right)=\frac{\partial \lambda_{2}}{\partial s}(1)=0$.

From Theorem 6.1, it is evident that the constructive algorithm presented in Section 5 can be adapted to identify a single point mass in a free-free beam. More precisely: the knowledge of the first and second eigenvalue allows to uniquely determine the intensity $m$ and the position $s$ of the point mass, up 
to a symmetric position with respect to the mid-point of the beam. We refer to Section 7.3 for an application to experimental data.

\subsection{Unique crack identification by two natural frequen- cies belonging to different spectra}

Let us denote by $\left\{\mu_{m}\right\}_{n=1}^{\infty}$ the eigenvalues of the cracked beam considered at the beginning of Section 6.1 when the boundary condition (50), at $x=0$, is replaced by

$$
u(0)=u^{\prime}(0)=0
$$

that is the left end of the beam is clamped. Therefore, by Proposition 2.2, the eigenvalues $\left\{\mu_{m}\right\}_{n=1}^{\infty}$ are those of a beam carrying a point mass $m=K^{-1}$ at $x=s$, with reversed end conditions:

$$
\left\{\begin{array}{l}
\left(b v^{\prime \prime}\right)^{\prime \prime}-\lambda r v=0, \quad \text { in }(0, s) \cup(s, 1), \\
\left(b v^{\prime \prime}\right)(0)=\left(b v^{\prime \prime}\right)^{\prime}(0)=0 \\
{[[v(s)]]=\left[\left[v^{\prime}(s)\right]\right]=\left[\left[\left(b v^{\prime \prime}\right)(s)\right]\right]=0} \\
{\left[\left[\left(b v^{\prime \prime}\right)^{\prime}(s)\right]\right]=\lambda m v(s)} \\
v(1)=v^{\prime}(1)=0
\end{array}\right.
$$

where $v=-a u^{\prime \prime}, b=\rho^{-1}$ and $r=a^{-1}$ in $[0,1]$.

By adapting the methods illustrated in preceding sections, under the symmetry assumption (18) for $b$ and $r$, and accepting a Vanishing Condition analogous to (20), we can prove the following result.

Theorem 6.2. For given $m, 0<m<\infty$, the first eigenvalue of (60)-(64) is a strictly increasing function of $s$, with $\mu_{1}(0)<\mu_{1}^{U}, \mu_{1}(1)=\mu_{1}^{U}, \frac{\partial \mu_{1}}{\partial s}(1)=0$. 
The main consequence of Theorem 6.2 is the following improvement of the identification result found in Section 5, namely: the knowledge of the first eigenvalue of the clamped-clamped beam and of the first eigenvalue of the free-clamped beam is enough for the unique determination of the mass location and intensity. Note that the symmetric solution is now avoided. The proof of this result can be obtained by adapting the reconstruction procedure illustrated in Section 5, see also Rubio et al. (2015, Section 7) for an analogous identification problem in a free-free longitudinally vibrating rod by one resonant frequency and one antiresonant frequency.

\section{Applications}

\subsection{Numerical approximation}

The practical application of the damage identification method requires the development of a specific numerical code. Fore the sake of simplicity and with the aim of illustrating the main aspects of the procedure, in the present section and in the next one reference is made to damage identification in a symmetric simply supported beam from the first two natural frequencies (see Section 5).

To find a finite element model of the weak formulation (22) of the eigenvalue problem (10)-(14), we work on the standard finite-dimensional subspace $\mathcal{H}^{N}$ of $\mathcal{H}$ (defined in (21)) formed by three-degree polynomial spline approximation of the transverse displacement of the beam axis. More precisely, let $\left\{x_{0}=0<x_{1}<x_{2}<\ldots<x_{N}<x_{N+1}=1\right\}$ be the nodes of a mesh of the interval $[0,1]$, with $x_{i+1}-x_{1}=\Delta x=\frac{1}{N+1}$, for every $i=0,1, \ldots, N$. 
The mesh is chosen such that there exists an index $i_{s} \in\{1, \ldots, N\}$ for which $s=i_{s} \Delta x$. Therefore, the discrete version of the eigenvalue problem (10)-(14) consists in finding the approximating eigenpair $(\widetilde{\lambda}, \widetilde{v}), \widetilde{v} \in \mathbb{R}^{2 N} \backslash\{0\}$, solution to

$$
K \widetilde{v}=\widetilde{\lambda} M \widetilde{v}
$$

where $K, M$ is the $2 N \times 2 N$ real symmetric matrix of the stiffness and of the inertia of the beam, respectively. The stiffness and mass matrix entries have been determined by integrating the exact expressions of the stiffness and mass density coefficients of the beam.

The numerical code of the reconstruction algorithm follows the steps shown in Section 5. In particular, a search strategy divided in two steps and having different size of the incremental mass step value $\Delta m$ has been implemented during the construction of the $\lambda$-s curves corresponding to increasing values of the point mass intensity. For the first iterations, we have used $\Delta m_{1}=k_{1} m_{1}^{-}$(case 1) and $\Delta m_{2}=k_{2} m_{2}^{-}$(case 2a and 2b, respectively), $k_{1}$ and $k_{2}$ being two positive numbers less than 1 to be chosen by the user. In the second stage, we have applied a constant incremental step $\Delta m_{f}$. In our experience, good choices are $k_{1}=k_{2}=\frac{1}{20}$, and $\Delta m_{f}=10^{-5}$ for all the case studied.

The reconstruction algorithm has been implemented on a computer with an Intel(R) Core (TM) i3 2.53 GHz processor and 4 GB of RAM. The whole procedure was built in Matlab environment. 


\subsection{Results of numerical simulations}

The identification algorithm has been tested on a large class of beam profiles having different intensity and position of the point mass. A selected, but representative, series of results is presented in the sequel for beams with (normalized) bending stiffness $a(x)$ and linear mass density $\rho(x)$ given by

$a_{S}(x)=(0.8-0.2 \sin (3 \pi x))^{3}, \quad \rho_{S}(x)=0.8-0.2 \sin (3 \pi x), \quad$ (sinusoidal profile)

$a_{P}(x)=(0.8-0.8 x(x-1))^{3}, \quad \rho_{P}(x)=0.8-0.8 x(x-1), \quad$ (parabolic profile)

$x \in[0,1]$. The approximating eigenvalue problem (65) was defined by dividing the interval $[0,1]$ into 200 equally spaced finite elements. The selected mesh size ensures negligible errors on the estimate of the first two natural frequencies of the beam.

Tables 1 and 2 collect the results of identification for various locations and intensities of the point mass. Specifically, ten equally spaced positions along the half-span and four intensities were considered. The values chosen for $m$ are associated to crack depth that may occur in practical situations (see also the experiments presented in the next section). For instance, the considered values for $m$ (from 0.01 to 0.5 ) correspond to percentage crack depth between 0.1 to 0.56 for a single transversal crack in a beam having rectangular cross-section and width-to-length ratio equal to 0.1.

It can be seen that the agreement between identified and actual values of the damage parameters is good. Generally speaking, some discrepancy emerged for cracks located near the end of the beam, which is known to be a point of vanishing sensitivity to damage for the natural frequencies 
of a simply supported beam. Moreover, errors are larger in case of small cracks (e.g., small values of $m$ ) and they decrease as $m$ increase. The typical computing time for each identification ranges from $30-100 \mathrm{~s}$ to $300-2200 \mathrm{~s}$ in case of $m=0.01$ and $m=0.50$, respectively, suggesting that the computation burden is larger in case of more severe levels of damage.

\subsection{Applications to experimental data}

With the aim of evaluating the stability of the method to errors on the data, an experimental application on a real cracked steel beam is presented in this section.

The mechanical model is a double $T$ free-free steel beam of the series $H E 100 B$. The length is $L=4 \mathrm{~m}$. The beam was suspended by means of two steel ropes so to simulate free-free boundary conditions. The damage was obtained by saw cutting the beam at progressive depth at the cross-section of abscissa $z_{d}=0.7 \mathrm{~m}$ far from the left end, see Figure 4 . Two damage configurations, denoted as D1 and D2 in the following, were realized. The width of each cut was equal to about $1.5 \cdot 10^{-3} \mathrm{~m}$ and, because of the small level of the excitation, during dynamic test the crack can be considered always open.

In order to measure the lower resonant frequencies of the beam, a modal analysis procedure based on impulsive excitation and measurement of the frequency response function (inertance) of the beam was adopted. Details of experiments can be found in Biscontin et al. (1998) and Caddemi and Morassi (2013). In brief, the transverse excitation was introduced at one end by means of an impulse force hammer, while the transverse response was 
measured by a piezoelectric accelerometer fixed at the same end of the beam. Vibration signals were acquired by a dynamic analyzer and then processed in the frequency domain to measure the relevant frequency response term. Natural frequencies were estimated by means of the classical single mode technique.

Table 3 compares the first two (positive) natural frequencies for the undamaged and damaged beam. For the sake of completeness, Table 3 also collects the theoretical values of the natural frequencies. Dynamic tests on the undamaged configuration were interpreted by means of the classical EulerBernoulli model, with mass density $\rho=20.775 \mathrm{kgm}^{-1}$ and bending stiffness $a=961421 \mathrm{Nm}^{-2}$. The mass density was evaluated from the total mass of the specimen under the hypothesis that the material is homogeneous, whereas the (constant) bending stiffness was calculated by matching the theoretical and the experimental values of the fundamental frequency. The analytical model of the cracked beam was defined by assuming the position $z_{d}$ of the damage as known and determining the theoretical value of the stiffness $K$ such that, for each damage configuration, the measured and the analytical fundamental frequency coincide. From Table 3 it emerges that, although the analytical model can be considered very accurate in the frequency range explored, the percentage experimental/modelling errors and the crack-induced frequency shifts of the damage configuration $D 1$ are comparable for the second frequency of the beam. In fact, frequency shifts with respect to the undamaged configuration of the first and second resonant frequency are equal to $-1.57,-6.19$ per cent in configuration $D 1$, respectively, whereas they are equal to $-7.05,-22.24$ per cent in configuration $D 2$. 
Table 4 collects the estimated values of the position of the cracked crosssection and the point mass. In the present case, identification is based on Theorem 6.1 and it has been performed by adapting the reconstruction algorithm shown in Section 5 to a beam under free-free end conditions. The agreement between actual and estimated values is good for configuration $D 2$, whereas, as expected, the inaccuracy of the data (particularly, on the second natural frequency) prejudices the identification of configuration $D 1$. The sensitivity of diagnostic techniques based on natural frequencies to experimental/modelling errors is a well-known pathology of the present class of inverse problems, and it has been pointed out by several authors, see, for example, Cerri and Vestroni (2000) and Dilena and Morassi (2004).

\section{A proof of the Vanishing Condition for small $m$}

In this section we show that the Vanishing Condition (20) is actually satisfied by the eigenfunctions of the problem (10)-(14) for small mass intensity $m$, that is, if $m$ is small enough, then the function $f_{m}^{(n)}$ defined in (19) has a finite number of zeros in $[0,1]$, all of which are simple zeros, $n \geq 1$. We provide a proof for a beam under pinned-pinned end conditions. Similar analysis can be developed for other sets of boundary conditions. Note that we are considering $n=1,2$.

Let $\left(\lambda_{n}, v_{n}\right)$ be the $n$th eigenpair of $(10)-(14)$, for positive functions $b, r$ satisfying the regularity conditions (17) and the symmetry conditions (18). Let $M>0$ be a finite given number and consider a point mass $m, m \in[0, M]$, 
located at the position $s \in[0,1]$. We find convenient to rewrite the function $f_{m}^{(n)}:[0,1] \rightarrow \mathbb{R}$ introduced in the proof of Theorem (4.5) as

$$
f_{m}^{(n)}(s)=V_{m}^{(n)}(s) D_{m}^{(n)}(s)
$$

where

$$
V_{m}^{(n)}(s)=\left.v_{n}(x ; s, m)\right|_{x=s}, \quad D_{m}^{(n)}(s)=\left.\frac{d v_{n}(x ; s, m)}{d x}\right|_{x=s} .
$$

The quantities $V_{m}^{(n)}(s)$ and $D_{m}^{(n)}(s)$ are, respectively, the value of the $n$th principal mode and the value of the $x$-derivative of the $n$th principal mode of the beam both evaluated at the cross-section in which the point mass $m$ is located. Hereinafter, in order to simplify the analysis, we shall assume the following normalization conditions

$$
m v_{n}^{2}(s ; s, m)+\int_{0}^{1} r(x) v_{n}^{2}(x ; s, m) d x=1,\left.\quad \frac{d v_{n}(x ; s, m)}{d x}\right|_{x=0}>0
$$

on the eigenfunction $v_{n}$ of $(10)-(14)$, for every $s \in[0,1]$ and every $m \in[0, M]$. It should be noticed that these conditions are not restrictive, since we are interested in studying the zeros of the function $f_{m}^{(n)}$.

Let us start by noticing that, by definition, the function $V_{m}^{(n)}$ vanishes at $s=0$ and $s=1$, and that $D_{m}^{(n)}(s) \neq 0$ at $s=0$ and $s=1$ (e.g., $D^{(n)}(0)=\left.\frac{d v_{n}(x ; 0, m)}{d x}\right|_{x=0}=\left(v_{n}^{U}\right)^{\prime}(0) \neq 0$, and similarly at $\left.s=1\right)$. Therefore, $s=0$ and $s=1$ are simple zeros of $f_{m}^{(n)}$. By the symmetry of the problem, it is easily seen that

$$
f_{m}^{(n)}(s)=-f_{m}^{(n)}(1-s), \quad s \in[0,1]
$$

therefore $s=\frac{1}{2}$ is another (simple) zero of $f_{m}^{(n)}$.

We shall present a proof in three steps: 
Step 1. The functions $V_{m}^{(n)}$ and $D_{m}^{(n)}$ do not vanish simultaneously.

Step 2. The zeros of $V_{m}^{(n)}$ are finite in number, and are all simple.

Step 3. The zeros of $D_{m}^{(n)}$ are finite in number, and are all simple.

In order to simplify the notation, hereinafter we omit the index $m$ in denoting the functions $V_{m}^{(n)}$ and $D_{m}^{(n)}$, and we shall simply write $V^{(n)}$ and $D^{(n)}$.

Proof of Step 1. In the sequel we restrict our attention to the open interval $(0,1)$. $V^{(1)}$ does not vanish in $(0,1)$. Then, if $z \in(0,1)$ is such that $V^{(2)}(z)=D^{(2)}(z)=0$, then $\left.v_{2}(x ; z, m)\right|_{x=z}=0$ and $\left.\frac{d v_{2}(x ; z, m)}{d x}\right|_{x=z}=0$, which is impossible since the zeros of $v_{2}$ and those of $v_{2}^{\prime}$ interlace by Proposition 3.1.

Proof of Step 2. Let us assume $n=2$.

We first show that the set of zeros of $V^{(n)}$ coincides with the set of zeros of $v_{n}^{U}$. Therefore, the zeros of $V^{(n)}$ are finite in number. If $V^{(n)}(z)=$ $v_{n}(z ; z, m)=0$ for certain $z \in(0,1)$, then $v_{n}^{U}(z)=0$ by Proposition 4.2 (point iv)). Conversely, if $v_{n}^{U}(z)=0$ for $z \in(0,1)$, then the function $v_{n}^{U}$ satisfies (10)-(14), with the point mass $m$ located at $z$ and the eigenvalue equal to $\lambda_{n}^{U}$. Then, $v_{n}^{U}$ is an eigenfunction of (10)-(14). Since $v_{n}^{U}$ has $n-1$ zeros in $(0,1)$, the function $v_{n}^{U}$ actually coincides with the $n$th eigenfunction $v_{n}$. Therefore, $0=v_{n}(z ; z, m)=V^{(n)}(z)$, and $z$ is a zero of $V^{(n)}$.

Let $z$ be a zero of $V^{(n)}$. We shall prove that, for $m$ small enough,

$$
\left.V^{(n)^{\prime}}(s)\right|_{s=z} \neq 0
$$

Assuming $V^{(n)}$ of $C^{1}$-class in $(0,1)$ and for a given $s_{0} \in(0,1)$, by direct calculation and using Lagrange's theorem with $0<\left|\alpha_{h}\right|<|h|$ and $0<\left|\beta_{h}\right|<$ 
$|h|$, we have

$$
\begin{gathered}
\left.V^{(n)^{\prime}}(s)\right|_{s=s_{0}}=\lim _{h \rightarrow 0} \frac{v_{n}\left(s_{0}+h ; s_{0}+h, m\right)-v_{n}\left(s_{0} ; s_{0}, m\right)}{h}= \\
=\lim _{h \rightarrow 0} \frac{v_{n}\left(s_{0}+h ; s_{0}+h, m\right)-v_{n}\left(s_{0} ; s_{0}+h, m\right)+v_{n}\left(s_{0} ; s_{0}+h, m\right)-v_{n}\left(s_{0} ; s_{0}, m\right)}{h}= \\
=\lim _{h \rightarrow 0}\left(\left.\frac{d v_{n}\left(x ; s_{0}+h, m\right)}{d x}\right|_{x=s_{0}+\alpha_{h}}+\left.\frac{d v_{n}\left(s_{0} ; s, m\right)}{d s}\right|_{s=s_{0}+\beta_{h}}\right)= \\
=\left.\frac{d v_{n}\left(x ; s_{0}, m\right)}{d x}\right|_{x=s_{0}}+\left.\frac{d v_{n}\left(s_{0} ; s, m\right)}{d s}\right|_{s=s_{0}} . \quad \text { (73) }
\end{gathered}
$$

Let us evaluate the partial derivative of the eigenfunction $v_{n}$ of (10)-(14) with respect to the position $s$ of the point mass $m$, under the normalization conditions (70). We begin with the incremental version of the variational formulation of the eigenvalue problem (22), precisely (here, we omit the dependence on $m$ and we write $(\cdot)^{\prime}=\frac{\partial(\cdot)}{\partial x}$ to further simplify the notation):

$$
\begin{aligned}
& \int_{0}^{1} b\left(\Delta_{h} v_{n}^{\prime \prime}\right) \varphi^{\prime \prime}=\Delta_{h} \lambda_{n}\left(m v_{n}(s ; s) \varphi(s)+\int_{0}^{1} r v_{n} \varphi\right)+ \\
& +\lambda_{n}\left[m\left(v_{n}^{\prime}\left(s+\zeta_{h} ; s+h\right) \varphi(s)+\left.\Delta_{h}\left(v_{n}(z ; s)\right)\right|_{z=s} \varphi(s)\right)+\right. \\
& \left.\quad+m v_{n}(s+h ; s+h) \Delta_{h}(\varphi(s))+\int_{0}^{1} r\left(\Delta_{h} v_{n}\right) \varphi\right]
\end{aligned}
$$

for every $\varphi \in \mathcal{H}=\left\{f \mid f \in H^{2}(0,1), f(0)=f(1)=0\right\}, 0<\zeta_{h}<|h|,|h|>0$ and $s \in[|h|, 1-|h|]$, where the operator $\Delta_{h}$ is defined as

$$
\Delta_{h} f(x ; s)=\frac{f(x ; s+h)-f(x ; s)}{h}, \quad h>0, s \in[0,1-h] .
$$

We look for the function $\Delta_{h} v_{n}$ in the form

$$
\Delta_{h} v_{n}=\sum_{j=1}^{\infty} a_{n j}^{(h)} v_{j}
$$

and we assume that the series is uniformly convergent in $[0,1]$, together with its derivatives with respect to $x$ up to the order 2 included. By replacing (76) 
in (74), choosing $\varphi=v_{l}\left(=v_{l}(x ; s, m)\right.$, with $s$ and $m$ given), and integrating by parts, we have

$$
\begin{aligned}
& \left(\lambda_{n}-\lambda_{l}\right) a_{n l}^{(h)}=-\Delta_{h} \lambda_{n} \delta_{n l}- \\
& \quad-\lambda_{n} m\left(v_{n}^{\prime}\left(s+\zeta_{h} ; s+h\right) v_{l}(s ; s)+\left.v_{n}(s+h ; s+h) \Delta_{h} v_{l}(x ; s)\right|_{x=s}\right),
\end{aligned}
$$

for every $l, l \geq 1$, and $n$. Taking $n=l$ and evaluating the limit of (77) as $h \rightarrow 0$ we obtain (31). If $n \neq l$, then we have

$$
a_{n l}^{(h)}=-\lambda_{n} m \frac{v_{n}^{\prime}\left(s+\zeta_{h} ; s+h\right) v_{l}(s ; s)+\left.v_{n}(s+h ; s+h) \Delta_{h} v_{l}(x ; s)\right|_{x=s}}{\left(\lambda_{n}-\lambda_{l}\right)} .
$$

By applying the incremental operator $\Delta_{h}$ to the normalization condition (70), we easily get

$$
a_{l l}^{(h)}=0, \quad \text { for every } l \geq 1 .
$$

Then, by inserting (78), (79) in (76), and taking the limit as $h \rightarrow 0$, we have:

$$
\begin{aligned}
& \frac{d v_{n}(x ; s, m)}{d s}= \\
= & -m \lambda_{n}(s ; m) \sum_{l=1, l \neq n}^{\infty} \frac{\left.\left(\frac{d v_{n}(x ; s, m)}{d x} v_{l}(x ; s, m)+v_{n}(x ; s, m) \frac{d v_{l}(x ; s, m)}{d x}\right)\right|_{x=s}}{\lambda_{n}(s, m)-\lambda_{l}(s, m)} \cdot v_{l}(x ; s, m) .
\end{aligned}
$$

If $z$ is a zero of $V^{(n)}$, then $v_{n}=v_{n}^{U}, \lambda_{n}=\lambda_{n}^{U}$ (see Proposition 4.2, proof of the point iv)), and $\frac{d v_{n}}{d s}$ at $(x=z ; s=z, m)$ takes the form

$$
\left.\frac{d v_{n}(z ; s, m)}{d s}\right|_{s=z}=-\left.m \lambda_{n}^{U} \frac{d v_{n}^{U}(x ; z, m)}{d x}\right|_{x=z} \sum_{l=1, l \neq n}^{\infty} \frac{v_{l}^{2}(z ; z, m)}{\lambda_{n}^{U}-\lambda_{l}(z, m)} .
$$

Replacing (81) in (73), and writing (73) for $s_{0}=z$ (where $z$ is a zero of $V^{(n)}$ ), we have

$$
\left.V^{(n)^{\prime}}(s)\right|_{s=z}=\left.\frac{d v_{n}^{U}(x ; z, m)}{d x}\right|_{x=z}\left(1+m \lambda_{n}^{U} \sum_{l=1, l \neq n}^{\infty} \frac{v_{l}^{2}(z ; z, m)}{\lambda_{l}(z, m)-\lambda_{n}^{U}}\right) .
$$


Now consider the beam in (10)-(14) with the point mass $m$ at $x=s$, and acted on by a concentrated transversal time-harmonic force $F=F_{0} \exp (i \sqrt{\lambda} t)$ at the point $x_{1}$ of the beam axis, with radian frequency $\sqrt{\lambda}$. The transversal deflection $v=v_{0} \exp (i \sqrt{\lambda} t)$ at the point $x_{2}$ is given by

$$
v_{0}=H_{F-v}\left(x_{1}, x_{2} ; \lambda\right) F_{0},
$$

where the function $H_{F-v}\left(x_{1}, x_{2} ; \lambda\right):[0,1] \times[0,1] \times\left(\mathbb{R} \backslash \bigcup_{l=1}^{\infty} \lambda_{l}(s, m)\right) \rightarrow \mathbb{R}$,

$$
H_{F-v}\left(x_{1}, x_{2} ; \lambda\right)=\sum_{l=1}^{\infty} \frac{v_{l}\left(x_{1} ; s, m\right) v_{l}\left(x_{2} ; s, m\right)}{\lambda_{l}(s, m)-\lambda},
$$

is the force-displacement receptance between the points $x_{1}$ and $x_{2}$, evaluated at $\lambda$. It follows that expression (82) can be written as

$$
\left.V^{(n)^{\prime}}(s)\right|_{s=z}=\left.\frac{d v_{n}^{U}(x ; z, m)}{d x}\right|_{x=z}\left(1+m \lambda_{n}^{U} \widetilde{H}_{F-v}\left(z, z ; \lambda_{n}^{U}\right)\right)
$$

where $\widetilde{H}_{F-v}$ means that the $n$th term of the series (84) of $H_{F-v}$ has been omitted. The function $\widetilde{H}_{F-v}(z, z ; \lambda)$ is well-defined at $\lambda=\lambda_{n}^{U}$ and, therefore, since $\left.\frac{d v_{n}^{U}(x ; z, m)}{d x}\right|_{x=z} \neq 0$ (e.g., the zeros of $v_{n}^{U}$ and those of $v_{n}^{U^{\prime}}$ interlace), condition (72) is satisfied for $m$ small enough.

Proof of Step 3. Let $n=1,2$. We start by proving that if all the zeros of $D^{(n)}$ are simple, then $D^{(n)}$ necessarily has a finite number of zeros in $(0,1)$. Let us assume that there exists an infinite family $\{z\}_{i=1}^{\infty}, z_{i} \in(0,1)$, such that $D^{(n)}\left(z_{i}\right)=0$ for every $i$. Then, on assuming $D^{(n)}$ regular enough, there exists $\zeta_{i}, z_{i-1}<\zeta_{i}<z_{i}$, for every $i \geq 2$, such that $D^{(n)^{\prime}}\left(\zeta_{i}\right)=0$. By BolzanoWeierstrass's theorem, there exists $\bar{z} \in(0,1)$ such that $\lim _{i \rightarrow \infty} z_{i}=\bar{z}$, and $D^{(n)}(\bar{z})=0$. Moreover, we also have $\lim _{i \rightarrow \infty} \zeta_{i}=\bar{z}$ and $D^{(n)^{\prime}}(\bar{z})=0$, and $\bar{z}$ is not a simple zero of $D^{(n)}$, a contradiction. 
To simplify the notation, let us write $\left.\frac{d v(x ; s, m)}{d x}\right|_{x=s}=\frac{d v}{d x}(s ; s)$. By direct calculation and by Lagrange's theorem, for every $s_{0} \in(0,1)$ and with $0<$ $\left|\gamma_{h}\right|<|h|, 0<\left|\delta_{h}\right|<|h|$, we have

$$
\begin{gathered}
\left.D^{(n)^{\prime}}(s)\right|_{s=s_{0}}=\lim _{h \rightarrow 0} \frac{\frac{d v_{n}}{d x}\left(s_{0}+h ; s_{0}+h\right)-\frac{d v_{n}}{d x}\left(s_{0} ; s_{0}\right)}{h}= \\
=\lim _{h \rightarrow 0} \frac{\frac{d v_{n}}{d x}\left(s_{0}+h ; s_{0}+h\right)-\frac{d v_{n}}{d x}\left(s_{0} ; s_{0}+h\right)+\frac{d v_{n}}{d x}\left(s_{0} ; s_{0}+h\right)-\frac{d v_{n}}{d x}\left(s_{0} ; s_{0}\right)}{h}= \\
=\lim _{h \rightarrow 0}\left(\frac{d^{2} v_{n}}{d x^{2}}\left(s_{0}+\gamma_{h} ; s_{0}+h\right)+\frac{d}{d s}\left(\frac{d v_{n}}{d x}\right)\left(s_{0} ; s_{0}+\delta_{h}\right)\right)= \\
=\left.\frac{d^{2} v_{n}\left(x ; s_{0}, m\right)}{d x^{2}}\right|_{x=s_{0}}+\left.\frac{d^{2} v_{n}\left(s_{0} ; s, m\right)}{d s d x}\right|_{s=s_{0}} . \quad
\end{gathered}
$$

On assuming sufficient regularity on $v_{n}$ with respect to the variables $x$ and $s$ (e.g., continuous second order partial derivatives), by Schwarz's theorem we have

$$
\frac{d^{2} v_{n}}{d s d x}=\frac{d}{d x}\left(\frac{d v_{n}}{d s}\right)
$$

where $\frac{d v_{n}}{d s}$ takes the expression (80). Then, deriving by series, we obtain

$$
\begin{aligned}
& \frac{d^{2} v_{n}(x ; s, m)}{d s d x}= \\
= & -m \lambda_{n} \sum_{l=1}^{\infty} \frac{\left.\left(\frac{d v_{n}(x ; s, m)}{d x} v_{l}(x ; s, m)+v_{n}(x ; s, m) \frac{d v_{l}(x ; s, m)}{d x}\right)\right|_{x=s}}{\lambda_{n}(s, m)-\lambda_{l}(s, m)} \cdot \frac{d v_{l}(x ; s, m)}{d x} .
\end{aligned}
$$

Let $z$ be a zero of $D^{(n)}$, e.g., $\left.\frac{d v_{n}(x ; z, m)}{d x}\right|_{x=z}=0$. Then, by (86) and (88) we have

$$
\left.D^{(n)^{\prime}}(s)\right|_{s=z}=\left.\frac{d^{2} v_{n}(x ; z, m)}{d x^{2}}\right|_{x=z}-m \lambda_{n}(z, m) v_{n}(z ; z, m) \sum_{l=1}^{\infty} \frac{\left(\left.\frac{d v_{l}(x ; z, m)}{d x}\right|_{x=n}\right)^{2}}{\lambda_{n}(z, m)-\lambda_{l}(z, m)},
$$

where $v_{n}(z ; z, m) \neq 0$ because the zeros of $v_{n}$ and those of $v_{n}^{\prime}$ interlace. 
As in Step 2, we consider the beam in (10)-(14) subject to a concentrated bending time-harmonic couple $C=C_{0} \exp (i \sqrt{\lambda} t)$ acting at the point $x_{1}$ of the beam axis. The rotation $\vartheta\left(=-\frac{d v}{d x}\right)$ of the transversal cross-section of the beam $\vartheta=\vartheta_{0} \exp (i \sqrt{\lambda} t)$ at the point $x_{2}$ is given by

$$
\vartheta_{0}=H_{C-v^{\prime}}\left(x_{1}, x_{2} ; \lambda\right) C_{0},
$$

where the function $H_{C-v^{\prime}}\left(x_{1}, x_{2} ; \lambda\right):[0,1] \times[0,1] \times\left(\mathbb{R} \backslash \bigcup_{l=1}^{\infty} \lambda_{l}(s, m)\right) \rightarrow \mathbb{R}$,

$$
H_{C-v^{\prime}}\left(x_{1}, x_{2} ; \lambda\right)=\sum_{l=1}^{\infty} \frac{v_{l}^{\prime}\left(x_{1} ; s, m\right) v_{l}^{\prime}\left(x_{2} ; s, m\right)}{\lambda_{l}(s, m)-\lambda},
$$

is the couple-rotation receptance between the points $x_{1}$ and $x_{2}$, evaluated at $\lambda$. Then, expression (89) becomes

$\left.D^{(n)^{\prime}}(s)\right|_{s=z}=\left.\frac{d^{2} v_{n}(x ; z, m)}{d x^{2}}\right|_{x=z}-m \lambda_{n}(z, m) v_{n}(z ; z, m) \widetilde{H}_{C-v^{\prime}}\left(z, z ; \lambda_{n}(z, m)\right)$,

where $\widetilde{H}_{C-v^{\prime}}$ is obtained from the corresponding $H_{C-v^{\prime}}$ by omitting the $n$th term. The function $\widetilde{H}_{C-v^{\prime}}(z, z ; \lambda)$ is well-defined at $\lambda=\lambda_{n}(z, m)$. Therefore, noting that $\left.\frac{d^{2} v_{n}(x ; z, m)}{d x^{2}}\right|_{x=z} \neq 0$ (e.g., the zeros of $v_{n}^{\prime}$ and those of $v_{n}^{\prime \prime}$ interlace), if $m$ is small enough, then $\left.D^{(n)^{\prime}}(s)\right|_{s=z} \neq 0$, and the zero is simple.

Remark 8.1. It should be noted that the result presented in this section holds only asymptotically, that is when $m$ is small enough or, equivalently, when the crack is sufficiently small. It would be useful to state the result from a quantitative point of view, namely by finding a value of the mass intensity in terms of the given data, say $m^{*}$, such that the Vanishing Condition (20) holds for $m \leq m^{*}$. 


\section{Conclusions}

This paper has been devoted to the inverse problem of identifying a single crack in a bending vibrating beam by minimal natural frequency data. The crack is assumed to remain open during vibration and it is modelled by a massless rotational linearly elastic spring located at the damaged crosssection.

We have established sufficient conditions for the unique identification of the crack location and severity in terms of a suitable pair of natural frequencies. The result is proved for a beam with symmetric smooth varying profile and has been derived without any assumption on the smallness of the crack severity. The methodology used for the proof leads to a constructive damage identification algorithm, and it is based on a careful analysis of the eigenvalues as functions of the damage position and damage intensity. $\mathrm{Nu}-$ merical results are in agreement with the theory when exact analytical data are employed in identification. Application to experimental data shows that the results of identification are rather sensitive to modelling errors.

Our result is proved under a technical a-priori assumption, namely the Vanishing Condition (20), on the zeros of a function determined in terms of the eigenfunctions associated to the natural frequencies used as data. It should be noted the Vanishing Condition (20) is actually a property of the eigenpairs of a simply supported beam with variable profile when the crack is small. In addition, by means of a different approach, we have recently shown in Fernández-Sáez et al. (2016) that the Vanishing Condition can be omitted when the simply-supported beam is uniform, without introducing any restriction on the damage severity. Whether the Vanishing Condition 
may or may not be definitively removed from the analysis of the inverse problem remains an open question, at the moment.

The method proposed in this paper may be used, in principle, also to deal with more general diagnostic problems for frame structures. Among possible applications, we mention the identification of a single crack in multispan beams (see also Vestroni and Capecchi (2000)) or frames (see Pau et al. (2011), Greco and Pau (2012) and Caddemi and Caliò (2013)), and the determination of multiple cracks (see Zheng and Fan (2001) and Caddemi and Caliò (2014)). However, it should be noted that some of the mathematical tools we have adopted in the treatment of the bending vibration of a single beam with a single crack have no straightforward generalization to those cases, and a preliminary analysis suggests that new ideas are needed to deal with these diagnostic problems.

\section{Acknowledgement}

The work of A. Morassi is partially supported by the University Carlos III of Madrid-Banco de Santander Chairs of Excellence Programme for the 2013-2014 Academic Year.

A. Morassi wishes to thank the colleagues of the University Carlos III of Madrid, especially Professors L. Rubio and J. Fernández-Sáez, for the warm hospitality at the Department of Engineering Mechanics.

\section{References}

Araujo Gomes AJM, Montalvao Silva JM (1991) Theoretical and experimental data on crack depth effects in the dynamic behavior of free-free beams. 
Proceedings 16th International Seminar on Modal Analysis, Florence, Italy, $15-24$.

Attar M (2012) A transfer matrix method for free vibration analysis and crack identification of stepped beams with multiple edge cracks and different boundary conditions. International Journal of Mechanical Sciences 57: 1933.

Biscontin G, Morassi A and Wendel P (1998) Asymptotic separation of the spectrum in notched rods. Journal of Vibration and Control 4(3): 237-251.

Brezis H (1986) Analisi Funzionale. Liguore Editore, Napoli, Italy.

Caddemi S and Caliò I (2013) The exact explicit dynamic stiffness matrix of multi-cracked Euler-Bernoulli beam and applications to damaged frame structures. Journal of Sound and Vibration 332(12): 3049-3063.

Caddemi S and Caliò I (2014) Exact reconstruction of multiple concentrated damages on beams. Acta Mechanica 225: 3137-3156.

Caddemi S and Morassi A (2013) Multi-cracked Euler-Bernoulli beams: mathematical modelling and exact solutions. International Journal of Solids and Structures 50(6): 944-956.

Cerri MN and Vestroni F (2000) Detection of damage in beams subjected to diffuse cracking. Journal of Sound and Vibration 234(2): 259-276.

Chaudhari TD and Maiti SK (2000) A study of vibration of geometrically segmented beams with and without crack. International Journal of Solids and Structures 37: 761-779. 
Chinchalkar S (2001) Determination of crack location in beams using natural frequencies. Journal of Sound and Vibration 247(3): 417-429.

Chondros TG and Dimarogonas AD (1980) Identification of cracks in welded joints of complex structures. Journal of Sound and Vibration 69(4): 531-538.

Courant R and Hilbert D (1966) Methods of Mathematical Physics (volume I), First English Edition, Interscience Publishers, Inc., New York.

Davini C, Gatti F and Morassi A (1993) A damage analysis of steel beams. Meccanica 28: 27-37.

Dilena M and Morassi A (2004) The use of antiresonances for crack detection in beams. Journal of Sound and Vibration 276(1-2): 195-214.

Fernández-Sáez J, Morassi A, Pressacco M and Rubio L (2016) The full nonlinear crack identification problem in a uniform simply supported beam in bending. Journal of Sound and Vibration 371: 94-109.

Freund LB and Herrmann G (1976) Dynamic fracture of a beam or plate in plane bending. Journal of Applied Mechanics 76-APM-15: 112-116.

Gladwell GML (2004) Inverse problems in vibration. Second edition, Kluwer Academic Publishers, Dordrecht, The Netherlands.

Greco A and Pau A (2012) Damage identification in Euler frames. Computer and Structures 92-93: 328-336.

Gudmundson P (1983) The dynamic behavior of slender structures with cross-sectional cracks. Journal of Mechanics and Physics of Solids 31(4): 329345 . 
Morassi A (1993) Crack-induced changes in eigenparameters of beam structures. Journal of Engineering Mechanics ASCE 119(9): 1798-1803.

Morassi A and Rovere N (1997) Localizing a notch in a steel frame from frequency measurements. Journal of Engineering Mechanics ASCE 123(5): 422-432.

Morassi A (2001) Identification of a crack in a rod based on changes in a pair of natural frequencies. Journal of Sound and Vibration 242(4): 577-596.

Nandwana BP and Maiti SK (1997) Detection of the location and size of a crack in stepped cantilever beams based on measurements of natural frequencies. Journal of Sound and Vibration 203(3): 435-446.

Narkis Y (1994) Identification of crack location in vibrating simply supported beams. Journal of Sound and Vibration 172(4): 549-558.

Pau A, Greco A and Vestroni F (2011) Numerical and experimental detection of concentrated damage in a parabolic arch by measured frequency variations. Journal of Vibration and Control 17(4): 605-614..

Pöschel J and Trubowitz E (1987) Inverse Spectral Theory, Academic Press, London, UK.

Rubio L (2009) An efficient method for crack identification in simply supported Euler-Bernoulli beams. Journal of Vibration and Acoustics 131, Paper 051001.

Rubio L, Fernández-Sáez J and Morassi A (2015) Identification of an open crack in a non-uniform rod by two frequency data. International Journal of Solids and Structures 75-76: 61-80. 
Shen M-HH and Taylor JE (1991) An indentification problem for vibrating cracked beams. Journal of Sound and Vibration 150(3): 457-484.

Sinha JK, Friswell MI and Edwards S (2002) Simplified models for the location of cracks in beam structures using measured vibration data. Journal of Sound and Vibration 251(1): 13-38.

Teughels A, Maeck J and De Roeck G (2002) Damage assessment by FE model updating using damage functions. Computer and Structures 80(25): $1869-1879$.

Vestroni F and Capecchi D (1996) Damage evaluation in cracked vibrating beams using experimental frequencies and finite element model. Journal of Vibration and Control 2(1): 69-86.

Vestroni F and Capecchi D (2000) Damage detection in beam structures based on frequency measurements. Journal of Engineering Mechanics ASCE 126: $761-768$.

Zheng DY and Fan SC (2001) Natural frequencies of a non-uniform beam with multiple cracks via modified Fourier series. Journal of Sound and Vibration 242(4): 701-717.

Weinberger HF (1965) A First Course in Partial Differential Equations, Dover Publications Inc., New York, US.

Wendtland D (1972) Änderung der Biegeeigenfrequenzen einer idealisierten Schaufel durch Risse. PhD Thesis, University of Karlsruhe. 


\section{Appendix}

In this section we collect the proofs of some of the statements made in Sections 2,3 and 4 .

\subsection{Proof of points 2. and 4. in Proposition 2.1}

In order to prove properties 2. and 4. stated in Proposition 2.1 it is enough to prove that the statical Green's function $G=G(x, \sigma):[0,1] \times[0,1] \rightarrow \mathbb{R}$ of the pinned-pinned Euler-Bernoulli cracked beam considered at the beginning of Section 3 is an oscillatory kernel, see, for instance, Gladwell (2004; Chapter 10 and Chapter 13).

We recall that the Green's function value $G(x, \sigma)$ is equal to the statical transversal deflection of the beam axis at $x$, when a transversal unit concentrated force is applied at the point of the beam axis of abscissa $\sigma$. A direct calculation shows that the Green's function for a pinned-pinned beam, having bending stiffness $a(x), a(x) \geq a_{0}>0$ in $[0,1]$ and $a \in C^{2}([0,1])$, and with a crack described by an elastic rotational spring of stiffness $K$ located at $s$ (see the jump conditions (3)-(4)), has the following expression.

For $\sigma \geq s:$

$$
\begin{array}{r}
G(x, \sigma)=\sigma x \int_{\sigma}^{1} \frac{(1-t)^{2}}{a(t)} d t+(1-\sigma)\left[(1-x) \int_{0}^{x} \frac{t^{2}}{a(t)} d t+x \int_{x}^{\sigma} \frac{t(1-t)}{a(t)} d t\right]+ \\
+\frac{x \sigma(1-\sigma)(1-s)}{K}, \quad x \in[0, s], \quad(93) \\
G(x, \sigma)=\sigma x \int_{\sigma}^{1} \frac{(1-t)^{2}}{a(t)} d t+(1-\sigma)\left[(1-x) \int_{0}^{x} \frac{t^{2}}{a(t)} d t+x \int_{x}^{\sigma} \frac{t(1-t)}{a(t)} d t\right]+ \\
+\frac{s^{2}(1-\sigma)(1-x)}{K}, \quad x \in[s, \sigma], \quad(94)
\end{array}
$$




$$
\begin{aligned}
G(x, \sigma)=(1-x)(1-\sigma) \int_{0}^{\sigma} \frac{t^{2}}{a(t)} d t & +\sigma\left[x \int_{x}^{1} \frac{(1-t)^{2}}{a(t)} d t+(1-x) \int_{\sigma}^{x} \frac{t(1-t)}{a(t)} d t\right]+ \\
& +\frac{s^{2}(1-\sigma)(1-x)}{K}, \quad x \in[\sigma, 1], \quad(95)
\end{aligned}
$$

and an analogous expression holds for $\sigma \leq s$. The Green's function is symmetric, e.g., $G(x, \sigma)=G(\sigma, x)$ for every $(x, \sigma) \in[0,1] \times[0,1]$. Note that $G$ is not defined for $K=0$.

Let us introduce some notation. Let $I$ be the open interval $(0,1)$ and, for every $n \geq 1$, let $Q=\left\{\mathbf{y}=\left(y_{1}, \ldots, y_{n}\right) \in \mathbb{R}^{n} \mid 0<y_{1}<\ldots<y_{n}<1\right\}$.

The kernel $G(x, \sigma)$ is oscillatory if the following three conditions are satisfied:

i) $G(x, \sigma)>0$, for every $x \in I$ and for every $\sigma \in I$;

ii) $G(\mathbf{x}, \sigma) \geq 0$, for every $\mathbf{x} \in Q$ and for every $\sigma \in Q$, where $\mathbf{x}=$ $\left(x_{1}, \ldots, x_{n}\right), \sigma=\left(\sigma_{1}, \ldots, \sigma_{n}\right)$ and

$$
G(\mathbf{x}, \sigma)=\operatorname{det}\left(\begin{array}{cccc}
G\left(x_{1}, \sigma_{1}\right) & \cdots & \cdots & G\left(x_{1}, \sigma_{n}\right) \\
G\left(x_{2}, \sigma_{1}\right) & \cdots & \cdots & G\left(x_{2}, \sigma_{n}\right) \\
\vdots & \vdots & \vdots & \vdots \\
G\left(x_{n}, \sigma_{1}\right) & \cdots & \cdots & G\left(x_{n}, \sigma_{n}\right)
\end{array}\right) .
$$

iii) $G(\mathbf{x}, \mathbf{x})>0$, for every $\mathbf{x} \in Q$.

Condition i) follows from the explicit expression (93)-(95) and from the analogous for $\sigma \leq s$.

To prove condition iii), let us assume that $n$ transversal concentrated forces $\left\{f_{i}\right\}_{i=1}^{n}$ are applied at points $\left\{x_{i}\right\}_{i=1}^{n}$ of the axis, $n$ finite and $n \geq 1$. By the linearity of the boundary value problem, the transversal displacement of the beam axis at $x_{j}$ is given by $u\left(x_{j}\right)=\sum_{i=1}^{n} G\left(x_{j}, x_{i}\right) f_{i}, j=1, \ldots, n$. The external work $W^{e}$ exerted by $\left\{f_{i}\right\}_{i=1}^{n}$ on the transversal deflection of the 
beam is equal to $W^{e}=\sum_{j=1}^{n} u\left(x_{j}\right) f_{j}=\sum_{i, j=1}^{n} G\left(x_{j}, x_{i}\right) f_{i} f_{j}$. By Clapeyron's theorem, $W^{e}$ is twice the strain energy stored in the cracked beam, namely

$$
\sum_{i, j=1}^{n} G\left(x_{j}, x_{i}\right) f_{i} f_{j}=\int_{0}^{1} \frac{M^{2}(x)}{a(x)} d x+\frac{M^{2}(s)}{K},
$$

where $M(x)=-a(x) u^{\prime \prime}(x)$ is the bending moment acting at the cross-section of abscissa $x$. From (97), the symmetric $n \times n$ matrix with entries $G_{j i}=$ $G\left(x_{j}, x_{i}\right), j, i=1, \ldots, n$, is positive definite. Therefore, $\operatorname{det}\left(G_{j i}\right)=G(\mathbf{x}, \mathbf{x})>$ 0 , for every $\mathrm{x} \in Q$.

The proof of the property ii) is more involved and, roughly speaking, it is based on the 'oscillatory' character of the transversal displacement $u(x)$ of the beam under the action of $n$ forces $\left\{f_{i}\right\}_{i=1}^{n}$. More precisely, it can be shown (see, for instance, Gladwell (2004), Chapter 10 and Chapter 13) that ii) holds true if the system has the following property.

Proposition 10.1. Let $\left\{f_{i}\right\}_{i=1}^{n}$ be a finite set of $n$ concentrated forces acting at points $\left\{\sigma_{i}\right\}_{i=1}^{n}$, respectively, with $0<\sigma_{1}<\ldots<\sigma_{n}<1, n \geq 1$. Then, the transversal displacement of the beam axis $u(x)$ has at most $(n-1)$ changes of sign in $(0,1)$.

The proof of Proposition 10.1 follows the lines used by Gladwell (2004, Theorem 13.2.6) to establish the analogous result for a beam without crack. Therefore, in the remaining of this section we only sketch the main steps of the proof, emphasizing the role played by the singularity in the analysis.

Following the arguments of the proof of Theorem 13.2.6 in (Gladwell, $2004)$, it is possible to prove that the second derivative $u^{\prime \prime}(x)$ of the transversal displacement $u(x)$ has at most $(n-1)$ changes in sign in $(0,1)$, say at $\left\{\xi_{i}\right\}_{i=1}^{n-1}$, with $0<\xi_{1}<\ldots<\xi_{n-1}<1$. Note that, since the bending moment 
$M(x)=-a(x) u^{\prime \prime}(x)$ is a piecewise-linear continuous function in $[0,1]$ (vanishing at the ends $x=0$ and $x=1$ ), actually each change of sign occurs in a node (simple zero) of $u^{\prime \prime}(x)$.

In order to estimate from above the zeros of $u^{\prime}(x)$, we need to consider the jump condition $K\left[\left[u^{\prime}(s)\right]\right]=a(s) u^{\prime \prime}(s)$ at the cracked cross-section. We distinguish two main cases, depending on whether $u^{\prime \prime}(s)$ vanishes or not.

If $u^{\prime \prime}(s)=0$, then the crack is not 'activated' by the forces $\left\{f_{i}\right\}_{i=1}^{n}$, and the function $u^{\prime}(x)$ is continuous in $[0,1]$. Therefore, we can use the results already available in (Gladwell, 2004) to conclude that $u^{\prime}(x)$ has at most $n$ nodes in $(0,1)$, say at $\left\{\eta_{i}\right\}_{i=1}^{n}$, with $0<\eta_{1}<\ldots<\eta_{n}<1$. Finally, taking the boundary conditions $u(0)=0=u(1)$ into account, one can show that $u(x)$ cannot change its sign in the end intervals $\left(0, \eta_{1}\right),\left(\eta_{n}, 1\right)$, hence $u(x)$ has at most $(n-1)$ changes of sign (nodes) in $(0,1)$.

Conversely, if $u^{\prime \prime}(s) \neq 0$, then there exists an index $j, j \in\{0, \ldots, n-1\}$, such that $s \in\left(\xi_{j}, \xi_{j+1}\right)$, where $\xi_{0}=0$ and $\xi_{n}=1$. The function $u^{\prime}(x)$ is a regular (continuous) function in $\left(0, \xi_{j}\right)$ and in $\left(\xi_{j+1}, 1\right)$. Therefore, we can apply standard arguments (see Gladwell (2004), Theorem 13.2.25) to prove that $u^{\prime}(x)$ has at most one change of sign (node) in each of the intervals $\left(0, \xi_{1}\right),\left(\xi_{1}, \xi_{2}\right), \ldots,\left(\xi_{j-1}, \xi_{j}\right),\left(\xi_{j+1}, \xi_{j+2}\right), \ldots,\left(\xi_{n-1}, 1\right)$, for a total of, at most, $j+(n-j-1)=n-1$ changes of sign.

To conclude the analysis for $u^{\prime}(x)$, it is enough to prove that there is at most one change of sign of $u^{\prime}(x)$ in $\left(\xi_{j}, \xi_{j+1}\right)$.

The proof is by contradiction. Suppose that there exist two points $\eta, \widetilde{\eta}$, with $\xi_{j}<\eta<\widetilde{\eta}<\xi_{j+1}$, such that $u^{\prime}(\eta)=u^{\prime}(\widetilde{\eta})=0$. If either $s<\eta$ or $s>\widetilde{\eta}$, then the function $u^{\prime}$ is regular in $(\eta, \widetilde{\eta})$ and, by Rolle's Theorem, there exists 
$z \in(\eta, \widetilde{\eta})$ such that $u^{\prime \prime}(z)=0$, contrary to hypothesis that $u^{\prime \prime}(x) \neq 0$ in $\left(\xi_{j}, \xi_{j+1}\right)$. It remains the case in which $\eta \leq s \leq \widetilde{\eta}$. Without loss of generality, we can assume $u^{\prime \prime}(x)>0$ in $\left(\xi_{j}, \xi_{j+1}\right)$. Integrating $u^{\prime \prime}(x)$ in $(\eta, s)$ and in $\left(s, \xi_{j+1}\right)$, respectively, we have

$$
\begin{gathered}
u^{\prime}\left(s^{-}\right)=u^{\prime}(\eta)+\int_{\eta}^{s} u^{\prime \prime}(t) d t=\int_{\eta}^{s} u^{\prime \prime}(t) d t>0 \\
u^{\prime}(x)=u^{\prime}\left(s^{+}\right)+\int_{s}^{x} u^{\prime \prime}(t) d t, \quad x \in\left(s, \xi_{j+1}\right) .
\end{gathered}
$$

We can determine $u^{\prime}\left(s^{+}\right)$from the jump condition $K\left[\left[u^{\prime}(s)\right]\right]=a(s) u^{\prime \prime}(s)$, namely

$$
u^{\prime}\left(s^{+}\right)=u^{\prime}\left(s^{-}\right)+\frac{a(s) u^{\prime \prime}(s)}{K},
$$

and by (98) and (100), we obtain $u^{\prime}\left(s^{+}\right)>0$. Evaluating (99) for $x=\widetilde{\eta}$ we get

$$
0=u^{\prime}(\widetilde{\eta})=u^{\prime}\left(s^{+}\right)+\int_{s}^{\widetilde{\eta}} u^{\prime \prime}(t) d t>0
$$

a contradiction.

Therefore, we have shown that $u^{\prime}(x)$ has at most $n$ changes (nodes) of sign in $(0,1)$.

To conclude, we need to estimate (from above) the number of zeros of $u(x)$.

The function $u^{\prime}(x)$ is a continuous function in $[0,1] \backslash\{s\}$, with a possible jump at $x=s$ (e.g., at the crack position). If $s$ coincides with one of the changes of sign of $u^{\prime}(x)$, say $s=\eta_{j}$ for some $j, j \in\{1, \ldots, n\}$, then we can use Corollary 13.2.2 in (Gladwell, 2004) to conclude that $u(x)$ has at most $(n-1)$ changes of sign (nodes) in $(0,1)$. Note that in this step we have used the end conditions $u(0)=0=u(1)$ to exclude that $u(x)$ can change of sign in the end intervals $\left(0, \eta_{1}\right),\left(\eta_{n}, 1\right)$. 
Therefore, we only need to discuss the case in which $s \in\left(\eta_{j}, \eta_{j+1}\right)$, for a certain index $j, j \in\{1, \ldots, n\}$. Without loss of generality, we can assume $u^{\prime}(x)>0$ in $\left(\eta_{j}, \eta_{j+1}\right)$. We proceed by contradiction as in the previous step. Suppose that $u(\zeta)=u(\widetilde{\zeta})=0$, with $\eta_{j}<\zeta<\widetilde{\zeta}<\eta_{j+1}$. If either $s<\zeta$ or $s>\widetilde{\zeta}$, the function $u(x)$ is regular in $(\zeta, \widetilde{\zeta})$ and, by Rolle's Theorem, there exists $\chi \in(\zeta, \widetilde{\zeta})$ such that $u^{\prime}(\chi)=0$, a contradiction. It remains the case $s \in(\zeta, \widetilde{\zeta})$. We have

$$
\begin{gathered}
u\left(s^{-}\right)=\int_{\zeta}^{s} u^{\prime}(t) d t>0 \\
u(x)=u\left(s^{+}\right)+\int_{s}^{x} u^{\prime}(t) d t, \quad x \in\left(s, \eta_{n+1}\right) .
\end{gathered}
$$

By the jump condition $[[u(s)]]=0$ we have $u\left(s^{-}\right)=u\left(s^{+}\right)$and then, evaluating (103) for $x=\widetilde{\zeta}$, we have

$$
0=u(\widetilde{\zeta})=u\left(s^{+}\right)+\int_{s}^{\widetilde{\zeta}} u^{\prime}(t) d t>0
$$

a contradiction.

Collecting the above results, we can conclude that $u(x)$ has at most $(n-$ 1) changes of sign (nodes) in $(0,1)$, and the proof of Proposition 10.1 is completed.

\subsection{Proof of Proposition 3.1}

Proof of statement 1. The function $y_{1}(x)=-v_{1}(x)=a(x) u_{1}^{\prime \prime}(x)$ belongs to $C^{2}(0,1)$ by $(10)$ and $(12)$. In particular, $y_{1}$ is a non trivial solution to

$$
\left\{\begin{array}{l}
y_{1}^{\prime \prime}=\lambda_{1} \rho u_{1}, \quad \text { in }(0,1) \\
y_{1}(0)=0=y_{1}(1)
\end{array}\right.
$$


where $u_{1}$ is the first eigenfunction of (1)-(5). By Proposition 2.1 (point 4.), $u_{1}$ does not vanish in $(0,1)$, for instance $u_{1}(x)>0$ in $(0,1)$. Then, the rightend side of (105) is positive in $[0,1]$ and, by the Maximum Principle (see, for instance, Weinberger (1965)), $y_{1}$ attains its maximum at the boundary of $[0,1]:$

$$
y_{1}(x) \leq 0, \quad \text { in }[0,1]
$$

Suppose that there exists $\chi \in(0,1)$ such that $y_{1}(\chi)=0$. Then, by Rolle's Theorem there exist $\chi^{-}, \chi^{+}, \chi^{-} \in(0, \chi), \chi^{+} \in(\chi, 1)$, such that $y_{1}^{\prime}\left(\chi^{-}\right)=$ $0=y_{1}^{\prime}\left(\chi^{+}\right)$. Again by Rolle's Theorem, there exists $\tilde{\chi} \in\left(\chi^{-}, \chi^{+}\right)$such that $y_{1}^{\prime \prime}(\widetilde{\chi})=0$, contrary to hypothesis $u_{1}(x)>0$ in $(0,1)$, and we can conclude that $y_{1}$ does not vanish in $(0,1)$.

Clearly, there exists at least one point $\xi \in(0,1)$ such that $y_{1}^{\prime}(\xi)=0$. We prove that this point is unique. By (105) and since $u_{1}$ does not change sign in $(0,1)$, the function $y^{\prime}$ is either a monotonically increasing function of $x$ (when $u_{1}>0$ in $\left.(0,1)\right)$ or a monotonically decreasing function of $x$ (when $u_{1}<0$ in $(0,1))$ in $(0,1)$. Therefore, in both cases, the point $\xi \in(0,1)$ at which $y_{1}^{\prime}$ vanishes is unique. The zero is obviously simple, since $y_{1}^{\prime \prime}(\xi) \neq 0$.

Proof of statement 2. By Proposition 2.2, the eigenfunction $v_{2}$ satisfies the orthogonality condition

$$
\int_{0}^{1} r(x) v_{1}(x) v_{2}(x) d x+m v_{1}(s) v_{2}(s)=0
$$

Therefore, since $v_{1}$ does not vanish in $(0,1)$ by previous statement 1 , the function $v_{2}$ has at least one change of sign in $(0,1)$. We shall prove that this change of sign is unique and that it corresponds to the unique simple zero (node) of $v_{2}$. Note that $v_{2} \in C^{2}([0,1])$. 
Suppose that there are two changes of sign of $v_{2}$ in $(0,1)$, say at $\chi_{1}, \chi_{2}$, with $0<\chi_{1}<\chi_{2}<1$. Then, by Rolle's Theorem, there exist points $\left\{\gamma_{i}\right\}_{i=1}^{3}$ such that $v_{2}^{\prime}\left(\gamma_{i}\right)=0, i=1,2,3$, with $\gamma_{1} \in\left(0, \chi_{1}\right), \gamma_{2} \in\left(\chi_{1}, \chi_{2}\right), \gamma_{3} \in\left(\chi_{2}, 1\right)$. Again by Rolle's Theorem, there exist $\eta_{1} \in\left(\gamma_{1}, \gamma_{2}\right)$ and $\eta_{2} \in\left(\gamma_{2}, \gamma_{3}\right)$ such that $v_{2}^{\prime \prime}\left(\eta_{1}\right)=v_{2}^{\prime \prime}\left(\eta_{2}\right)=0$, namely $v_{2}^{\prime \prime}$ has at least two zeros in $(0,1)$. This is a contradiction, since $v_{2}^{\prime \prime}=-\lambda_{2} \rho u_{2}$ and, by Proposition 2.1, $u_{2}$ has only one (simple) zero in $(0,1)$. Therefore, $v_{2}$ has exactly one change of sign in $(0,1)$, say at $\xi_{1} \in(0,1)$. Using again Rolle's Theorem, one can show that $v_{2}(x) \neq 0$ in $(0,1)$ for $x \neq \xi_{1}$ and $\xi_{1}$ is the unique zero of $v_{2}$ in $(0,1)$. Finally, if $v_{2}^{\prime}\left(\xi_{1}\right)=0$, there exist $\tilde{\chi} \in\left(0, \xi_{1}\right), \widehat{\chi} \in\left(\xi_{1}, 1\right)$ such that $v_{2}^{\prime \prime}(\widetilde{\chi})=v_{2}^{\prime \prime}(\widehat{\chi})=0$, again a contradiction. Therefore, $\xi_{1}$ is simple.

Since $v_{2}$ has exactly one simple zero in $(0,1)$, say $v_{2}\left(\xi_{1}\right)=0$, there exist at least two points in $(0,1)$ at which $v_{2}^{\prime}=0$. Using Rolle's Theorem it can be shown that there are exactly two points $\eta_{1}, \eta_{2} \in(0,1)$ such that $v_{2}^{\prime}\left(\eta_{1}\right)=v_{2}^{\prime}\left(\eta_{2}\right)=0$, with $0<\eta_{1}<\xi_{1}<\eta_{2}<1$. Moreover, since $\int_{0}^{1} v_{2}^{\prime}=0$, $v_{2}^{\prime}$ must change sign (at least once) in $(0,1)$, Without loss of generality, we can assume that $v_{2}^{\prime}$ changes sign at $\eta_{1}$ and vanishes at $\eta_{2}$. It is easy to see that $\eta_{1}$ and $\eta_{2}$ are simple zeros of $v_{2}^{\prime}$ (that is, there is a change in sign also in $\eta_{2}$ ). If $\eta_{2}$ is not a simple zero of $v_{2}^{\prime}$, then $v_{2}^{\prime \prime}\left(\eta_{2}\right)=0$. Since $v_{2}^{\prime}\left(\eta_{1}\right)=0=v_{2}^{\prime}\left(\eta_{2}\right)$, there exits $\bar{\eta} \in\left(\eta_{1}, \eta_{2}\right)$ such that $v_{2}^{\prime \prime}(\bar{\eta})=0$, and $v_{2}^{\prime \prime}$ has two distinct zeros in $(0,1)$, a contradiction. Using similar considerations, one can prove that $\eta_{1}$ is a simple zero of $v_{2}^{\prime}$. Therefore, the function $v_{2}^{\prime}$ has two simple zeros in $(0,1)$ at $\eta_{1}$ and at $\eta_{2}$, and $\eta_{1}<\xi_{1}<\eta_{2}$. 


\section{Table Captions}

Table 1. Identification of the mass intensity $m$ and position $s$ in a simplysupported non-uniform beam with the sinusoidal profile given in (66), by the first two natural frequencies. Percentage errors: $e_{m}=100 \times\left(m_{e s t}-m\right) / m$, $e_{s}=100 \times\left(s_{e s t}-s\right) / s$.

Table 2. Identification of the mass intensity $m$ and position $s$ in a simplysupported non-uniform beam with the parabolic profile given in (67), by the first two natural frequencies. Percentage errors: $e_{m}=100 \times\left(m_{e s t}-m\right) / m$, $e_{s}=100 \times\left(s_{\text {est }}-s\right) / s$.

Table 3. Experimental test: first two (positive) natural frequencies $f_{n}=\frac{\omega_{n}}{2 \pi}$, $n=1,2$, (expressed in $\mathrm{Hz}$ ) of the undamaged free-free beam and their values associated to damage configurations $D_{i}, i=1,2$. Note: Modelling errors $\Delta$ are reported in brackets, $\Delta=100 \times\left(f_{n}^{\text {Model }}-f_{n}^{E x p}\right) / f_{n}^{E x p}$.

Table 4. Identification results based on experimental data: actual values $(s, m)$ versus estimated values $\left(s_{\text {est }}, m_{\text {est }}\right)$. Percentage errors in brackets: $100 \times\left(s_{\text {est }}-s\right) / s, 100 \times\left(m_{\text {est }}-m\right) / m . t=$ computing time (in seconds). 


\section{Figure Captions}

Figure 1. The $\lambda$-curves identification algorithm based on first two resonant frequencies: first case.

Figure 2. The $\lambda$-curves identification algorithm based on first two resonant frequencies: second case, subcase a).

Figure 3. The $\lambda$-curves identification algorithm based on first two resonant frequencies: second case, subcase b).

Figure 4. Experimental model and damage configurations. Lengths in millimeters. 
Table 1: Identification of the mass intensity $m$ and position $s$ in a simplysupported non-uniform beam with the sinusoidal profile given in (66), by the first two natural frequencies. Percentage errors: $e_{m}=100 \times\left(m_{\text {est }}-m\right) / m$, $e_{s}=100 \times\left(s_{e s t}-s\right) / s$.

\begin{tabular}{cccccccccccccc}
\hline & \multicolumn{3}{c}{$\mathrm{m}=0.01$} & \multicolumn{4}{c}{$\mathrm{m}=0.05$} & \multicolumn{3}{c}{$\mathrm{m}=0.20$} & \multicolumn{3}{c}{$\mathrm{m}=0.50$} \\
\cline { 2 - 13 } $\mathrm{s}$ & $e_{m}$ & $e_{s}$ & Case & $e_{m}$ & $e_{s}$ & Case & $e_{m}$ & $e_{s}$ & Case & $e_{m}$ & $e_{s}$ & Case \\
\hline 0.03 & -8.90 & 4.63 & $2 \mathrm{~b}$ & -0.05 & 0.03 & $2 \mathrm{~b}$ & -3.09 & 1.51 & $2 \mathrm{~b}$ & -1.77 & 0.85 & $2 \mathrm{~b}$ \\
0.08 & 1.77 & -0.98 & $2 \mathrm{~b}$ & 0.14 & -0.08 & $2 \mathrm{~b}$ & -0.11 & 0.06 & $2 \mathrm{~b}$ & 0.01 & -0.01 & $2 \mathrm{~b}$ \\
0.13 & 0.04 & -0.02 & $2 \mathrm{~b}$ & -0.01 & 0.01 & $2 \mathrm{~b}$ & 0.00 & 0.00 & $2 \mathrm{~b}$ & 0.00 & 0.00 & $2 \mathrm{~b}$ \\
0.18 & 0.07 & -0.06 & $2 \mathrm{~b}$ & 0.02 & -0.02 & $2 \mathrm{~b}$ & 0.00 & 0.00 & $2 \mathrm{~b}$ & 0.00 & 0.00 & $2 \mathrm{~b}$ \\
0.23 & 0.03 & -0.07 & $2 \mathrm{~b}$ & 0.01 & -0.02 & $2 \mathrm{~b}$ & 0.00 & -0.01 & $2 \mathrm{~b}$ & 0.00 & -0.01 & $2 \mathrm{~b}$ \\
0.28 & 0.02 & 0.04 & $2 \mathrm{a}$ & 0.00 & 0.01 & $2 \mathrm{a}$ & 0.00 & 0.00 & $2 \mathrm{a}$ & 0.00 & 0.00 & $2 \mathrm{a}$ \\
0.33 & 0.07 & 0.03 & $2 \mathrm{a}$ & 0.01 & 0.01 & $2 \mathrm{a}$ & 0.00 & 0.00 & $2 \mathrm{a}$ & 0.00 & 0.00 & 1 \\
0.38 & 0.04 & 0.01 & 1 & 0.01 & 0.00 & 1 & 0.00 & 0.00 & 1 & 0.00 & 0.00 & 1 \\
0.43 & 0.03 & 0.00 & 1 & 0.01 & 0.00 & 1 & 0.00 & 0.00 & 1 & 0.00 & 0.00 & 1 \\
0.48 & 0.05 & 0.00 & 1 & 0.00 & 0.00 & 1 & 0.00 & 0.00 & 1 & 0.00 & 0.00 & 1 \\
\hline
\end{tabular}


Table 2: Identification of the mass intensity $m$ and position $s$ in a simplysupported non-uniform beam with the parabolic profile given in (67), by the first two natural frequencies. Percentage errors: $e_{m}=100 \times\left(m_{\text {est }}-m\right) / m$, $e_{s}=100 \times\left(s_{e s t}-s\right) / s$.

\begin{tabular}{cccccccccccccc}
\hline & \multicolumn{3}{c}{$\mathrm{m}=0.01$} & \multicolumn{4}{c}{$\mathrm{m}=0.05$} & \multicolumn{3}{c}{$\mathrm{m}=0.20$} & \multicolumn{3}{c}{$\mathrm{m}=0.50$} \\
\cline { 2 - 13 } $\mathrm{s}$ & $e_{m}$ & $e_{s}$ & Case & $e_{m}$ & $e_{s}$ & Case & $e_{m}$ & $e_{s}$ & Case & $e_{m}$ & $e_{s}$ & Case \\
\hline 0.03 & -34.90 & 24.06 & $2 \mathrm{~b}$ & -4.44 & 2.18 & $2 \mathrm{~b}$ & -2.21 & 1.06 & $2 \mathrm{~b}$ & 0.43 & -0.23 & $2 \mathrm{~b}$ \\
0.08 & 0.35 & -0.19 & $2 \mathrm{~b}$ & -0.39 & 0.21 & $2 \mathrm{~b}$ & 0.02 & -0.01 & $2 \mathrm{~b}$ & 0.02 & -0.01 & $2 \mathrm{~b}$ \\
0.13 & 0.12 & -0.08 & $2 \mathrm{~b}$ & 0.02 & -0.01 & $2 \mathrm{~b}$ & 0.00 & 0.00 & $2 \mathrm{~b}$ & 0.00 & 0.00 & $2 \mathrm{~b}$ \\
0.18 & 0.08 & -0.08 & $2 \mathrm{~b}$ & 0.02 & -0.02 & $2 \mathrm{~b}$ & 0.00 & 0.00 & $2 \mathrm{~b}$ & 0.00 & 0.00 & $2 \mathrm{~b}$ \\
0.23 & 0.09 & -0.21 & $2 \mathrm{~b}$ & 0.01 & -0.04 & $2 \mathrm{~b}$ & 0.00 & 0.00 & $2 \mathrm{~b}$ & 0.00 & 0.00 & $2 \mathrm{a}$ \\
0.28 & 0.06 & 0.08 & $2 \mathrm{a}$ & 0.02 & 0.02 & $2 \mathrm{a}$ & 0.00 & 0.00 & $2 \mathrm{a}$ & 0.00 & 0.00 & $2 \mathrm{a}$ \\
0.33 & 0.04 & 0.02 & $2 \mathrm{a}$ & 0.00 & 0.00 & $2 \mathrm{a}$ & 0.00 & 0.00 & 1 & 0.00 & 0.00 & 1 \\
0.38 & 0.07 & 0.01 & 1 & 0.02 & 0.00 & 1 & 0.00 & 0.00 & 1 & 0.00 & 0.00 & 1 \\
0.43 & 0.05 & 0.00 & 1 & 0.01 & 0.00 & 1 & 0.00 & 0.00 & 1 & 0.00 & 0.00 & 1 \\
0.48 & 0.10 & 0.00 & 1 & 0.01 & 0.00 & 1 & 0.00 & 0.00 & 1 & 0.00 & 0.00 & 1 \\
\hline
\end{tabular}


Table 3: Experimental test: first two (positive) natural frequencies $f_{n}=\frac{\omega_{n}}{2 \pi}$, $n=1,2$, (expressed in $\mathrm{Hz}$ ) of the undamaged free-free beam and their values associated to damage configurations $D_{i}, i=1,2$. Note: Modelling errors $\Delta$ are reported in brackets, $\Delta=100 \times\left(f_{n}^{\text {Model }}-f_{n}^{\text {Exp }}\right) / f_{n}^{\text {Exp }}$.

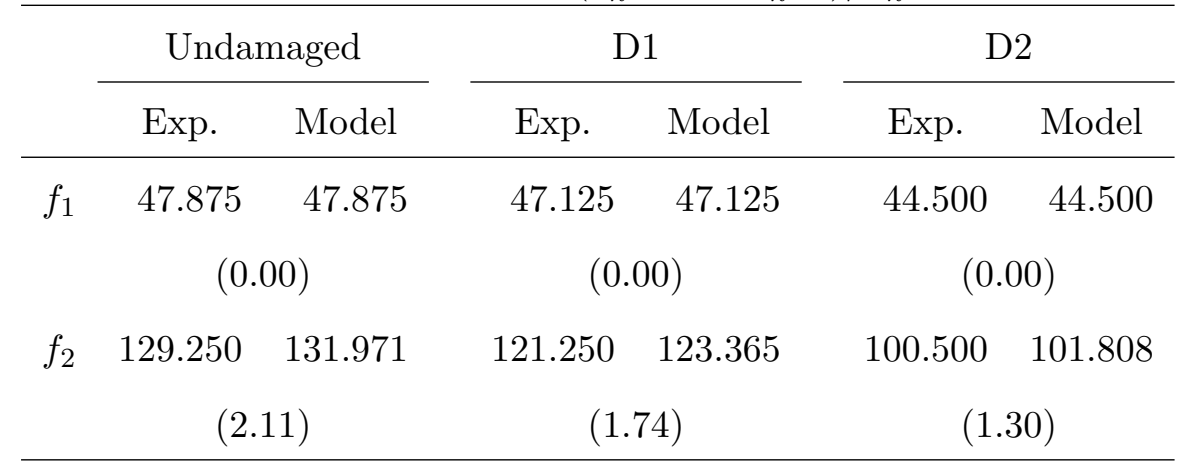


Table 4: Identification results based on experimental data: actual values $(s, m)$ versus estimated values $\left(s_{\text {est }}, m_{\text {est }}\right)$. Percentage errors in brackets: $100 \times\left(s_{\text {est }}-s\right) / s, 100 \times\left(m_{\text {est }}-m\right) / m . t=$ computing time (in seconds).

\begin{tabular}{ccc} 
& $\mathrm{D} 1$ & $\mathrm{D} 2$ \\
\cline { 2 - 3 }$s$ & 0.1750 & 0.1750 \\
$m$ & 0.1241 & 0.5475 \\
\hline$s_{\text {est }}$ & $0.1366(-21.96)$ & $0.1671(-4.51)$ \\
$m_{\text {est }}$ & $0.2816(126.96)$ & $0.6311(15.26)$ \\
\hline$t$ & 670.18 & 1076.26
\end{tabular}




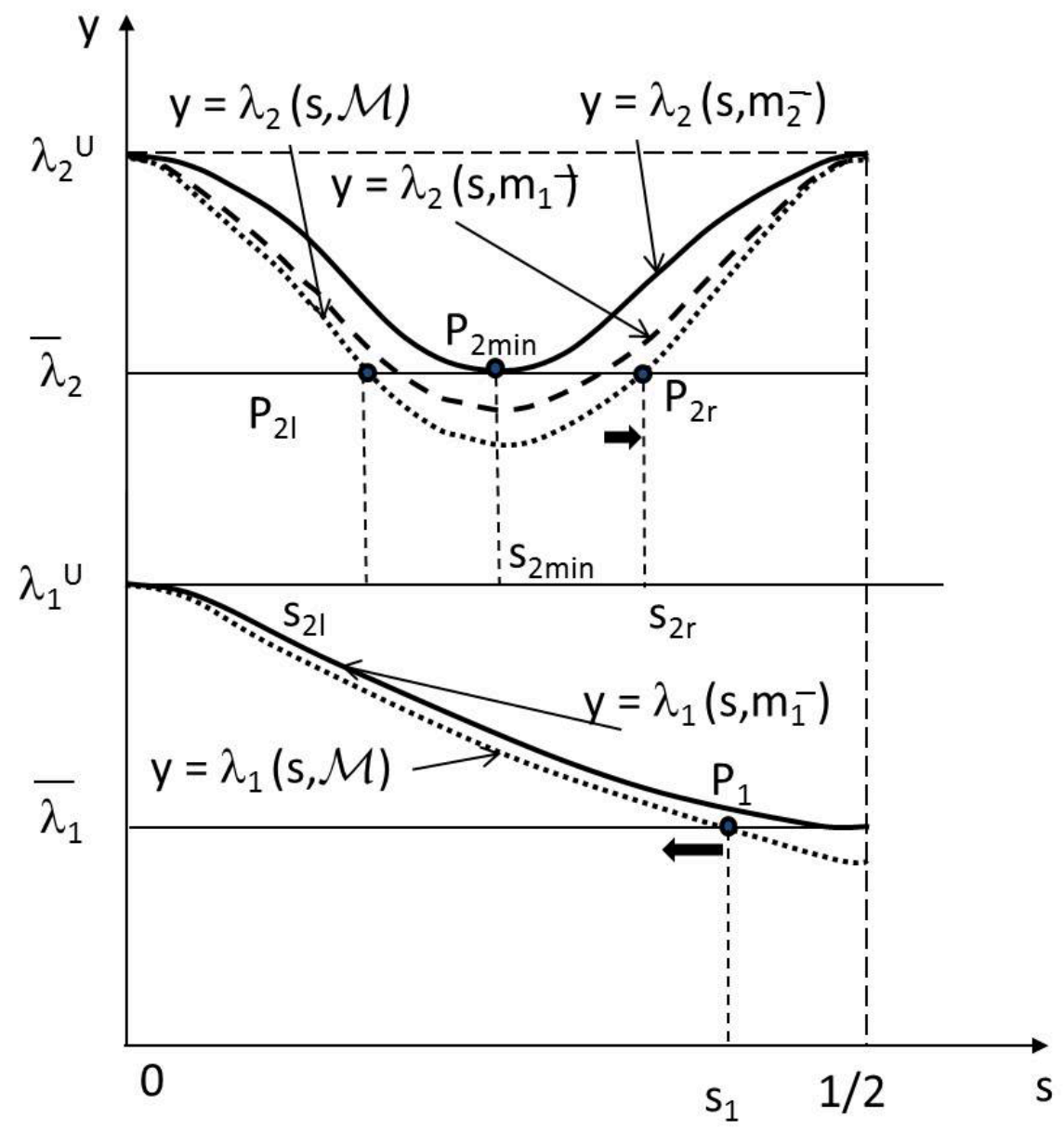

Figure 1: The $\lambda$-curves identification algorithm based on first two resonant frequencies: first case. 


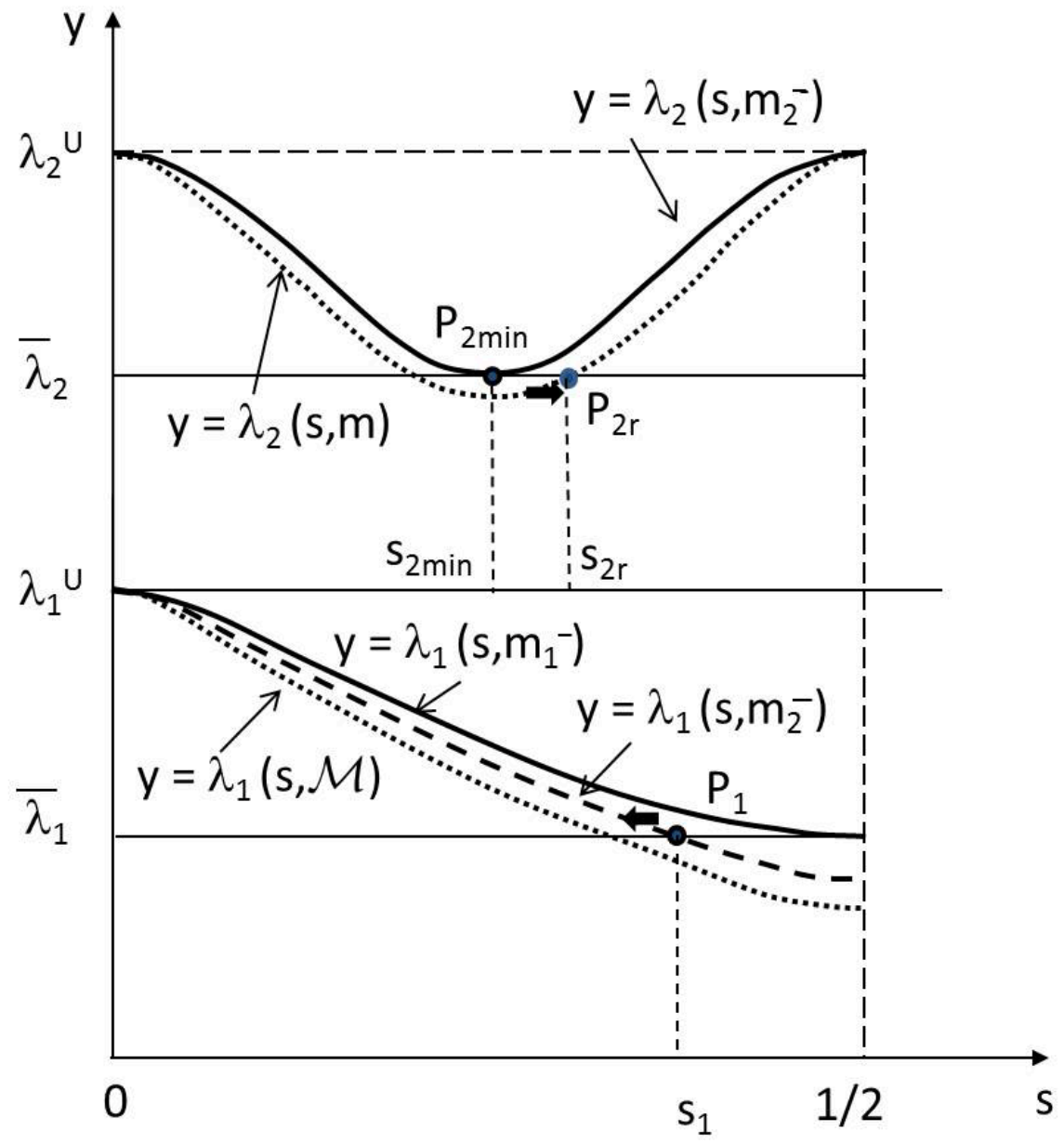

Figure 2: The $\lambda$-curves identification algorithm based on first two resonant frequencies: second case, subcase a). 


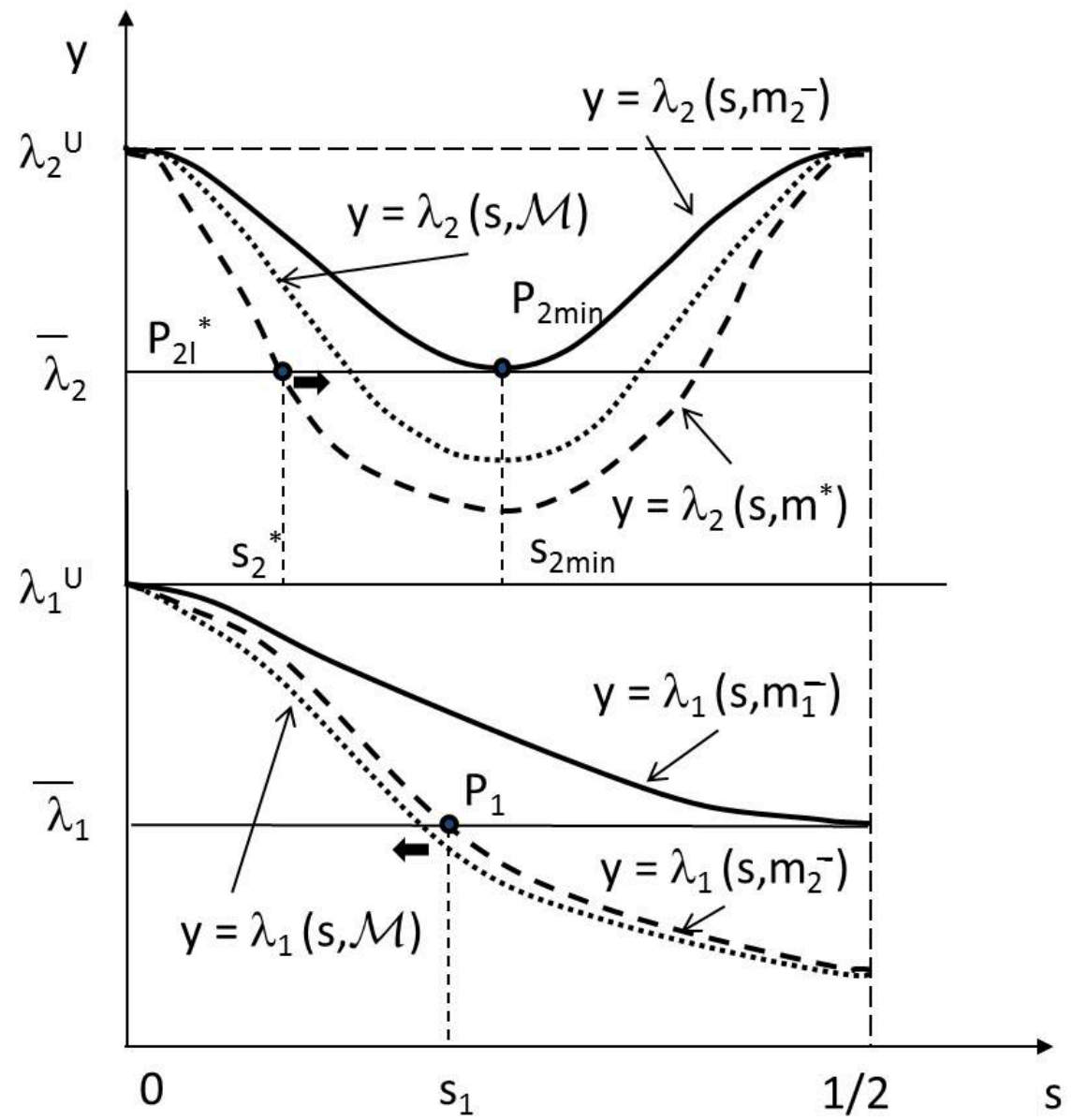

Figure 3: The $\lambda$-curves identification algorithm based on first two resonant frequencies: second case, subcase b). 

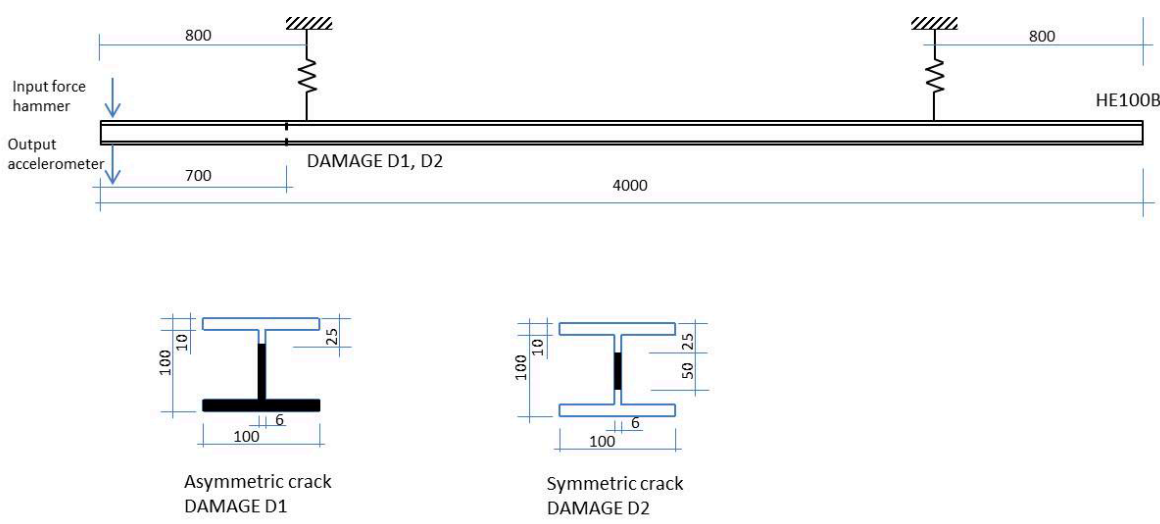

Figure 4: Experimental model and damage configurations. Lengths in millimeters. 